\title{
Induced order and collective excitations in three-singlet quantum magnets
}

\author{
Peter Thalmeier \\ Max Planck Institute for the Chemical Physics of Solids, D-01187 Dresden, Germany
}

(Received 23 January 2021; revised 25 March 2021; accepted 5 April 2021; published 29 April 2021)

\begin{abstract}
The quantum magnetism in a three-singlet model (TSM) with singlet crystalline electric field states interacting on a lattice is investigated, motivated by its appearance in compounds with $4 f^{2}$ and $5 f^{2}$ electronic structures. Contrary to conventional (semiclassical) magnetism, there are no preformed moments above the ordering temperature $T_{m}$. They appear spontaneously as induced or excitonic moments due to singlet-singlet mixing at $T_{m}$. In most cases, the transition is of second order, however, for large matrix elements between the excited states, it turns into a first-order transition at a critical point. Furthermore, we derive the excitonic mode spectrum and its quantum critical soft mode behavior, which leads to the criticality condition for induced order as expressed in terms of the control parameters of the TSM, and discuss the distinctions to the previously known two-singlet case. We also derive the temperature dependence of order parameters for second- and first-order transitions and the exciton spectrum in the induced magnetic phase.
\end{abstract}

DOI: 10.1103/PhysRevB.103.144435

\section{INTRODUCTION}

In ordinary (semiclassical) magnets, the individual magnetic moments at every lattice site already exist above the ordering temperature $T_{m}$ [1]. This holds even in strongly frustrated local-moment systems which may have a vanishing ordering temperature when fine-tuned to a spin-liquid regime where quantum fluctuations destroy the moment of the ground state but nevertheless the Curie-Weiss signature of local moments remains for elevated temperatures [2-4]. There are, however, true quantum magnets which do not have freely rotating magnetic moments above $T_{m}$ in the semiclassical sense as witnessed by an absence of the Curie-Weiss-type susceptibility for some region above $T_{m}$. In these compounds with partly filled $4 f$ or $5 f$ electron shells, the degenerate ground state with integer (non-Kramers) total angular momentum $J$, created by spin-orbit coupling splits due to the local crystalline electric field (CEF) into a series of multiplets [5]. They belong to irreducible representations $\Gamma_{i}$ which may comprise singlets, doublets, or triplets, depending on the symmetry of the $\mathrm{CEF}$ and the concrete $\mathrm{CEF}$ potential. For tetragonal or lower symmetry, it is possible that the ground state and lowest excited states are all singlets without magnetic moments, meaning $\left\langle\Gamma_{i}|\mathbf{J}| \Gamma_{i}\right\rangle=0$. Nevertheless, magnetic order occurs below the transition temperature $T_{m}$. This order cannot be interpreted in the usual semiclassical way as an alignment of preexisting moments which then have collective semiclassical spin-wave excitations as Goldstone modes. In the latter case,

Published by the American Physical Society under the terms of the Creative Commons Attribution 4.0 International license. Further distribution of this work must maintain attribution to the author(s) and the published article's title, journal citation, and DOI. Open access publication funded by the Max Planck Society. quantum effects enter only through the possible reduction of the saturation moment due to zero point fluctuations leading to spin-wave contribution to the ground-state energy.

For CEF systems with split-singlet low-lying states, the local moments instead appear only simultaneously with the magnetic order as a true quantum effect due to the mixing of singlet states caused by intersite exchange interactions. This induced or excitonic magnetic order has been observed primarily in various $\operatorname{Pr}\left(4 f^{2}\right)$ and $\mathrm{U}\left(5 f^{2}\right)$ compounds with two $f$ electrons, which lead to CEF schemes with singlet ground states and possibly also low-energy excited singlets. However, it can also be found in $f$-electron compounds with even higher $f$ occupations, like, e.g., $\mathrm{Tb}\left(4 f^{8}\right)$. In the cubic $\left(O_{h}\right)$ symmetry cases with singlet ground states, the excited states must be degenerate as in fcc $\operatorname{Pr}$ [6,7], $\mathrm{PrSb}$ [8], $\mathrm{Pr}_{3} \mathrm{Tl}$ [9], and $\mathrm{TbSb}$ [10] (singlet-triplet). Examples with hexagonal $\left(D_{6 h}\right)$ structures are metallic $\operatorname{Pr}$ (singlet-doublet) [5,11-13] and $\mathrm{UPd}_{2} \mathrm{Al}_{3}$ [14] (singlet-singlet). Tetragonal $\left(D_{4 h}\right)$ cases are $\mathrm{Pr}_{2} \mathrm{CuO}_{4}$ [15] (singlet-doublet) and $\mathrm{URu}_{2} \mathrm{Si}_{2}$ [16-18] (three singlets). The lower the symmetry, the more likely one can have multiple low-energy singlets. The most promising class in this respect has orthorhombic symmetry $\left(D_{2 h}, D_{2}\right)$ which has only singlets left as in $\mathrm{PrCu}_{2}$ [19,20], $\mathrm{PrNi}[21,22], \mathrm{Tb}_{3} \mathrm{Ga}_{5} \mathrm{O}_{12}$ [23], and $\mathrm{Pr}_{5} \mathrm{Ge}_{4}$ [24]. In the $\mathrm{U}$ compounds, however, the situation may be more complicated due to only partial localization of $5 f$ electrons $[25,26]$. Since there is no degeneracy in the local $4 f$ or $5 f$ basis states, there can also be no continuous symmetry for the exchange Hamiltonian. Therefore, the collective excitations in the ordered phase may not be interpreted as spin waves resulting from coupled local spin precessions but rather as dispersive singlet-singlet (or singlet-doublet and singlettriplet) excitation modes due to intersite exchange, commonly termed magnetic excitons. These are already present above the ordering temperature. The ordering is characterized by a softening of one of these modes at $T_{m}$ and a subsequent stiffening again further below. 
This type of excitonic magnetism has been considered analytically primarily within the two-singlet model $[5,14,27,28]$. A fully numerical treatment for a multilevel CEF system is also possible [29]. However, for a deeper understanding of induced excitonic moment ordering and their finite temperature properties, analytical investigations are desirable. In particular, the influence of physical parameters like splittings, nondiagonal matrix elements, and exchange, which define dimensionless control parameters on the transition temperature, saturation moment, and mode softening are rendered understandable only when explicit analytical expressions can be derived. This becomes quite involved beyond the twosinglet model. The latter is, however, an oversimplificiation as very often more levels, in particular, another singlet state are present, as, e.g,. in $\mathrm{PrNi}, \mathrm{PrCu}_{2}$, and $\mathrm{URu}_{2} \mathrm{Si}_{2}$.

Therefore, in this paper, we give a detailed analytical treatment of induced moment behavior in the physically important three-singlet model (TSM) relevant for non-Kramers $f$-electron systems in lower than cubic symmetry; in particular; we investigate the case of orthorhombic symmetry. We will focus on the mode spectrum, transition temperature, and saturation moments and how they are influenced by the larger set of control parameters of this extended model. We show that under suitable conditions, temperature variation induces hybridization of exciton modes in addition to the changes of intensity pattern. Furthermore, we derive an algebraic equation that completely determines the transition temperature for the effective two control parameters and arbitrary splitting ratio of the TSM. In the symmetric TSM, explicit closed expressions for $\mathrm{T}_{m}$ are presented. Furthermore, we give a comparative treatment of the exciton mode dispersions within random phase approximation (RPA) response function formalism and Bogoliubov quasiparticle picture and show that they give largely equivalent results, also for the phase boundary between disordered and excitonic phases. Finally, within the RPA formalism, we will investigate the change of mode dispersions and intensity in the induced moment phase. This paper is mainly theoretically motivated with the aim to analyze and understand the TSM and its significance for excitonic magnetism in detail.

\section{THE THREE-SINGLET MODEL}

We keep the specifications of the TSM illustrated in the inset of Fig. 1 as general as possible, as far as splittings and magnetic matrix elements of $J_{z}$ are concerned. However, having orthorhombic CEF systems in mind, the latter are assumed to be of uniaxial character due to $\left\langle\Gamma_{i}\left|J_{x, y}\right| \Gamma_{j}\right\rangle=0$ [Sec. II A, Eq. (4)]. The three singlets are denoted by $|i\rangle(i=1-3)$ with increasing level energies $E_{i}=0, \Delta, \Delta_{0}$, or shifted energies $\hat{E}_{i}=E_{i}-\Delta=-\Delta, 0, \tilde{\Delta}$, which are more convenient for finite-temperature properties; here we defined $\tilde{\Delta}=\Delta_{0}-\Delta$. The CEF Hamiltonian in can be written in terms of standard basis operators $L_{i j}=|i\rangle\langle j|$ as

$$
H_{\mathrm{CEF}}=\sum_{i} E_{i}|i\rangle\langle i| .
$$

The total angular momentum component $J_{z}$ in this representation is given by $J_{z}=\sum_{i j}\left\langle i\left|J_{z}\right| j\right\rangle L_{i j}$. Without restriction, this leaves us with three possible independent matrix elements

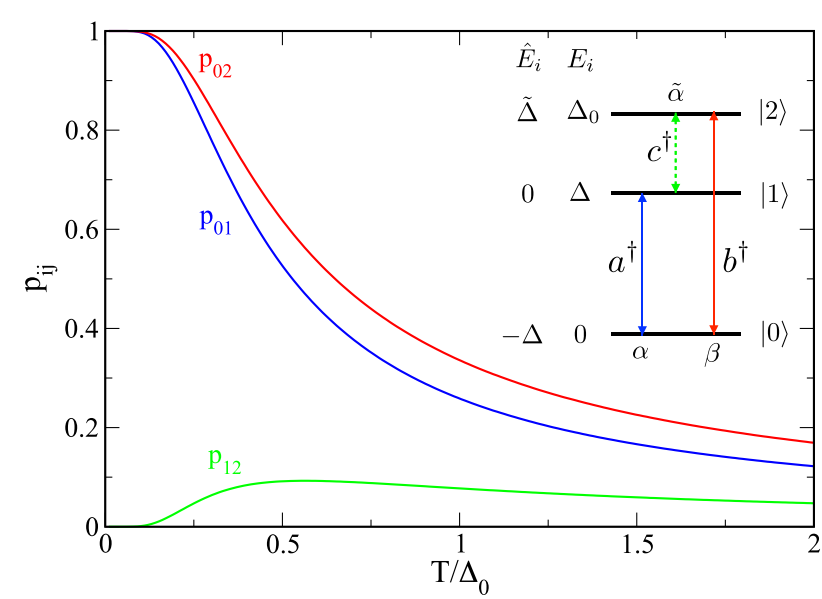

FIG. 1. Inset: Designations of the general TSM with singlet states $|i\rangle(i=1-3)$ and respective energies $E_{i}$ or $\hat{E}_{i}=E_{i}-\Delta$, here $\tilde{\Delta}=\Delta_{0}-\Delta$. Matrix elements of $J_{z}$ are denoted by $\alpha, \beta, \tilde{\alpha}$ (one of them must vanish in the nonmagnetic state) and boson excitation operators by $a^{\dagger}, b^{\dagger}, c^{\dagger}$. The special fully symmetric TSM is defined by $r=\tilde{\Delta} / \Delta=1$ and $\tilde{\alpha}=\alpha$. Transition arrows correspond to the thermal occupation differences $p_{i j}$ shown in the main figure. Here $r=0.5$, the energy scale is $\Delta_{0}$ in all figures.

$\alpha=\left\langle 0\left|J_{z}\right| 1\right\rangle, \beta=\left\langle 0\left|J_{z}\right| 2\right\rangle$, and $\tilde{\alpha}=\left\langle 1\left|J_{z}\right| 2\right\rangle$ (Sec. II A). The latter only plays a role at finite temperature $T$ when excited states are populated with population numbers $p_{i}=$ $Z^{-1} \exp \left(-E_{i} / T\right)$ where $Z=\sum_{j} \exp \left(-E_{j} / T\right)$ is the threesinglet partition function. The $N_{f}=3 \mathrm{CEF}$ wave functions may each be gauged by an arbitrary phase factor $\exp \left(i \phi_{n}\right)$ $\left(n=1 . . N_{f}\right)$. Furthermore, there are $\frac{1}{2} N_{f}\left(N_{f}-1\right)=3$ excitation matrix elements between those states. Therefore, in the TSM, all matrix elements $\alpha, \beta, \tilde{\alpha}$ may be chosen as real without loss of generality. We will pay particular attention to the special case of the (fully) symmetric TSM which is defined by $\tilde{\Delta}=\Delta ; \tilde{\alpha}=\alpha$ in Fig. 1 . The magnetic properties of the model are characterized by the three possible dimensionless control parameters,

$$
\xi_{\alpha}=\frac{2 \alpha^{2} I_{e}}{\Delta}, \quad \xi_{\beta}=\frac{2 \beta^{2} I_{e}}{\Delta_{0}}, \quad \xi_{\tilde{\alpha}}=\frac{2 \tilde{\alpha}^{2} I_{e}}{\tilde{\Delta}},
$$

which characterize the intersite-coupling strengths of the three transitions with $I_{e}(\mathbf{q})$ denoting the Fourier transform of the intersite exchange in Eqs. (9) and (10) at the wave vector of incipient induced order where it is at maximum value. It may be $\mathbf{q}=0$ ferromagnetic (FM), general incommensurate or $\mathbf{q}=\mathbf{Q}=(\pi, \pi, \pi)$ antiferromagnetic (AF). In the following, we focus on the latter case. As we shall see now in the paramagnetic phase, one of the three matrix elements or control parameters must vanish due to the requirements of time-reversal symmetry. This leaves us with the three possible cases of TSMs depicted in Fig. 2.

\section{A. The orthorhombic three-singlet model}

To realize the general TSM in a concrete CEF for given $J$ is not so straightforward as it may seem. This is connected with the angular momentum structure of CEF eigenstates and their behavior under time reversal. As already indicated in 
(a)

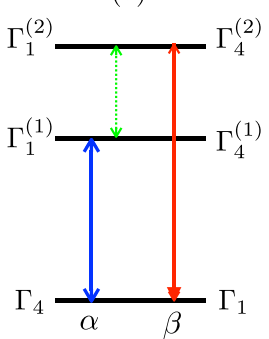

(b)

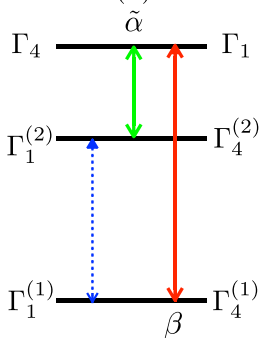

(c)

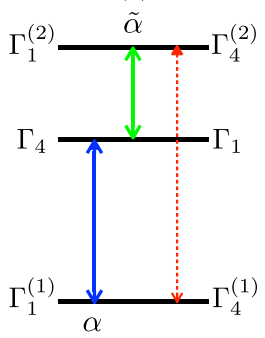

FIG. 2. Three possible arrangements of dipolar $\left(J_{z}\right)$ transition matrix elements $(\alpha, \beta, \tilde{\alpha})$ in the orthorhombic TSM consisting of $\left(\Gamma_{1}, \Gamma_{4}^{(1,2)}\right)$ or $\left(\Gamma_{4}, \Gamma_{1}^{(1,2)}\right)$ representations. In the paramagnetic phase, only two elements (thick lines) can be nonzero due to $\Theta$ invariance. Transitions with dashed lines vanish because they connect states with equal $\Theta$; they are, however, induced and nonzero in the magnetic state. Similar diagrams hold for the representation pair $\left(\Gamma_{2}, \Gamma_{3}\right)$ [cf. Eq. (4)]. There always have to be two (inequivalent) representations of the same type in the TSM for the $J_{z}$ structure presented.

the Introduction, cubic symmetry does not allow the TSM. In tetragonal $D_{4 h}$ symmetry, the TSM is only realized in one specific form [Fig. 2(c)] (see discussion in Appendix A). Therefore, we relax to orthorhombic symmetry $D_{2 h}$, where all the CEF states have to be singlet $\Gamma_{i},(i=1-4)$ representations and the TSM is naturally possible. As mentioned before, there are several physical realizations in the orthorhombic symmetry class. The decomposition, e.g., for $J=4$ leads to nine $\left(3 \Gamma_{1} \oplus 2 \Gamma_{2} \oplus 2 \Gamma_{3} \oplus 2 \Gamma_{4}\right)$ singlets. They may be grouped according to their behavior under time-reversal symmetry operation $\Theta[30]$. For a CEF state $|\psi\rangle$ written as linear combination $|\psi\rangle=\sum_{M} c_{M}|M\rangle(|M| \leqslant J)$ for a given $J$, the action of $\Theta$ is defined as $|\psi\rangle^{K}=\Theta|\psi\rangle=\sum_{M} c_{M}^{*}(-1)^{J-M}|-M\rangle$. The orthorhombic singlets for $J=4$ may be expressed as [23] linear combinations of $|M\rangle_{ \pm}=|M\rangle \pm|-M\rangle$ with only real coefficients according to

$$
\begin{aligned}
& \left|\Gamma_{1}\right\rangle=\sum_{M=0,2,4} b_{M}|M\rangle_{+}, \quad\left|\Gamma_{2}\right\rangle=\sum_{M=1,3} a_{M}^{\prime}|M\rangle_{-}, \\
& \left|\Gamma_{3}\right\rangle=\sum_{M=1,3} a_{M}|M\rangle_{+}, \quad\left|\Gamma_{4}\right\rangle=\sum_{M=2,4} b_{M}^{\prime}|M\rangle_{-} .
\end{aligned}
$$

This means that $\left|\Gamma_{1,2}\right\rangle^{K}=\left|\Gamma_{1,2}\right\rangle$ are even $\Theta=1$ ) and $\left|\Gamma_{3,4}\right\rangle^{K}=-\left|\Gamma_{3,4}\right\rangle$ are odd $(\Theta=-1)$ under time reversal. Because $J_{z}$ is also odd, it has matrix elements only among singlets with opposite $\Theta$. From those, only two are different from zero:

$$
\begin{aligned}
& \left\langle\Gamma_{1}\left|J_{z}\right| \Gamma_{4}\right\rangle=2 \sum_{M=2,4} M b_{M} b_{M}^{\prime}, \\
& \left\langle\Gamma_{2}\left|J_{z}\right| \Gamma_{3}\right\rangle=2 \sum_{M=1,3} M a_{M} a_{M}^{\prime} .
\end{aligned}
$$

At the same time, one observes that $\left\langle\Gamma_{1}\left|J_{x, y}\right| \Gamma_{4}\right\rangle=$ $\left\langle\Gamma_{2}\left|J_{x, y}\right| \Gamma_{3}\right\rangle=0$ so we can restrict to $J_{z}$ in the model for intersite interactions (Sec. III B). If the singlet representations in the TSM would all be different, then only one matrix element of $J_{z}$ could be nonzero. However, in the $J=4 D_{4 h}$ decomposition given above, each singlet representation occurs at least twice. Then two matrix elements of the TSM containing two singlets with equal symmetry can be nonzero. Because these have necessarily equal $\Theta$, the third $J_{z}$ matrix element is always zero as long as time-reversal symmetry holds. In the induced magnetic phase when $\Theta$ is broken, it will also be nonzero as shown in Appendix B [Eq. (B7)]; this is essential to obtain the proper temperature dependence of order parameter and soft mode energy.

In the paramagnetic phase, we are then left with the three possible cases of dipolar matrix element sets $(\alpha, \beta, \tilde{\alpha})$ as illustrated in Fig. 2. Since the orthorhombic CEF is characterized by nine arbitrary CEF parameters, one may reasonably expect that every sequence in Fig. 2 and similar ones with $\left(\Gamma_{2}, \Gamma_{3}\right)$ singlets can in principle be realized. We note that in the higher $D_{4 h}$ symmetry, only the model type of Fig. 2(c) seems possible (Appendix A). Rather than discussing each possible case presented in Fig. 2 individually, it is more economic to treat the general TSM (inset of Fig. 1), keeping in mind that always one in the set of matrix elements $(\alpha, \beta, \tilde{\alpha})$ must vanish to reproduce any of the possible cases in Fig. 2 allowed by $\Theta$.

\section{RESPONSE FUNCTION FORMALISM, MAGNETIC EXCITON BANDS, AND INDUCED TRANSTION}

\section{A. Local dynamic susceptibility of the TSM}

The most direct way to understand the magnetic ordering in the TSM is provided by the response function formalism, the resulting magnetic exciton bands, and their soft-mode behavior. The dynamic response function for the isolated TSM is, in general, given by

$$
\chi_{0}\left(i \omega_{n}\right)=\sum_{i j} \frac{\left|\left\langle i\left|J_{z}\right| j\right\rangle\right|^{2}\left(p_{j}-p_{i}\right)}{E_{i}-E_{j}-i \omega_{n}},
$$

defining the occupation differences of levels by $p_{i j}=p_{i}-p_{j}$. This is evaluated explicitly as $\left(\Delta_{0}=\Delta+\tilde{\Delta}\right)$,

$\chi_{0}\left(i \omega_{n}\right)=2\left[\frac{\alpha^{2} \Delta p_{01}}{\Delta^{2}-\left(i \omega_{n}\right)^{2}}+\frac{\tilde{\alpha}^{2} \tilde{\Delta} p_{12}}{\tilde{\Delta}^{2}-\left(i \omega_{n}\right)^{2}}+\frac{\beta^{2} \Delta_{0} p_{02}}{\Delta_{0}^{2}-\left(i \omega_{n}\right)^{2}}\right]$,

where the $p_{i j}$ are given by

$$
p_{01}=\frac{\tanh \frac{\Delta}{2 T}}{1-f_{01}}, \quad p_{02}=\frac{\tanh \frac{\Delta_{0}}{2 T}}{1+f_{02}}, \quad p_{12}=\frac{\tanh \frac{\tilde{\Delta}}{2 T}}{1+f_{12}},
$$

with

$$
\begin{aligned}
& f_{01}=\frac{1}{2}\left(\cosh \frac{\tilde{\Delta}}{T}-\sinh \frac{\tilde{\Delta}}{T}\right)\left(\tanh \frac{\Delta}{2 T}-1\right), \\
& f_{02}=\frac{1}{2}\left(\cosh \frac{\Delta}{T}-\sinh \frac{\Delta}{T}\right)\left(\tanh \frac{\Delta_{0}}{2 T}+1\right), \\
& f_{12}=\frac{1}{2}\left(\cosh \frac{\Delta}{T}+\sinh \frac{\Delta}{T}\right)\left(\tanh \frac{\tilde{\Delta}}{2 T}+1\right) .
\end{aligned}
$$

The occupation differences fulfill the relation $p_{12}=p_{02}-$ $p_{01}$. For $T \ll \Delta, \Delta_{0}$ when $p_{01}, p_{02} \leqslant 1$, this means $p_{12} \ll$ $p_{01}, p_{01}$. For the $t w o$-singlet model $(i, j=0,1)$, one simply has $p_{i j}=\tanh \frac{\beta}{2} \Delta_{i j}$. In the TSM, the expressions $f_{i j}$ in the denominators of Eq. (7) are a correction, taking into account the presence of the third level in the partition function. 


\section{B. Collective magnetic exciton modes}

The relevant part of the intersite exchange interaction of three-singlet states is given by $\left(l, l^{\prime}\right.$ denote lattice sites $\left.\mathbf{R}_{l}, \mathbf{R}_{l}^{\prime}\right)$

$$
\begin{aligned}
H_{\mathrm{ex}} & =-\frac{1}{2} \sum_{l l^{\prime}} I_{l l^{\prime}} J_{z}(l) J_{z}\left(l^{\prime}\right) \\
& =-\frac{1}{2} \sum_{\mathbf{q}} I_{e}(\mathbf{q}) J_{z}^{\mathbf{q}} J_{z}^{-\mathbf{q}},
\end{aligned}
$$

with the Fourier component $J_{z}^{\mathbf{q}}=N^{-\frac{1}{2}} \sum_{l} \exp \left(i \mathbf{q} \mathbf{R}_{l}\right) J_{z}^{l}$. The transverse $J_{x}^{\mathbf{q}}, J_{y}^{\mathbf{q}}$ do not contribute to the collective mode dispersion because of their vanishing matrix elements in the orthorhombic TSMs of Fig. 2. The Fourier transform of the exchange interaction may be expressed (assuming only next neighbor coupling $I_{0}$ ) as

$$
I_{e}(\mathbf{q})=2 I_{0} \gamma_{\mathbf{q}}, \quad \gamma_{\mathbf{q}}=\sum_{n=1}^{D} \cos q_{n}
$$

in the simple orthorhombic lattice of dimension $D=3$ and coordination $z=2 D$. The momentum units are $\frac{1}{a}, \frac{1}{b}$, and $\frac{1}{c}$ parallel to the respective orthogonal axes. For the AF case with $I_{0}<0$ on which we focus, we also introduce the effective AF exchange $I_{e} \equiv I_{e}(\mathbf{Q})=-z I_{0}>0$ where $\mathbf{Q}=(\pi, \pi, \pi)$ denotes the AF wave vector. Then, within RPA approximation [5], the collective dynamic susecptiblity ( $z z$ component only) of coupled three singlet levels is obtained as

$$
\chi\left(\mathbf{q}, i \omega_{n}\right)=\left[1-I_{e}(\mathbf{q}) \chi_{0}\left(i \omega_{n}\right)\right]^{-1} \chi_{0}\left(i \omega_{n}\right) .
$$

Its poles, as defined by $1-I_{e}(\mathbf{q}) \chi_{0}\left(i \omega_{n}\right)=0$, give the dispersive collective magnetic exciton modes of the TSM which are determined by a cubic equation in $\omega^{2}$. We first derive its general solution and then a more intuitive restricted one for the low-temperature case.

If we had only one of each contribution in Eq. (6), we would obtain isolated exciton modes given by

$$
\begin{aligned}
\omega_{e}^{2}(\mathbf{q}, T) & =\Delta\left[\Delta-2 \alpha_{T}^{2} I_{e}(\mathbf{q})\right], \\
\omega_{e^{\prime}}^{2}(\mathbf{q}, T) & =\Delta_{0}\left[\Delta_{0}-2 \beta_{T}^{2} I_{e}(\mathbf{q})\right], \\
\omega_{e^{\prime \prime}}^{2}(\mathbf{q}, T) & =\tilde{\Delta}\left[\tilde{\Delta}-2 \tilde{\alpha}_{T}^{2} I_{e}(\mathbf{q})\right],
\end{aligned}
$$

where we used the effective $T$-dependent transition strengths defined by

$$
\alpha_{T}^{2}=\alpha^{2} p_{01}, \quad \beta_{T}^{2}=\beta^{2} p_{02}, \quad \tilde{\alpha}_{T}^{2}=\alpha^{2} p_{12} .
$$

These uncoupled modes are hybridized into new eigenmodes when more matrix elements are present. We already derive these expressions in sight of the magnetic case of Sec. VIB where all the $\alpha_{T}^{\prime 2}, \beta_{T}^{2}, \tilde{\alpha}_{T}^{\prime 2}$ as modified by the molecular field are nonzero. The hybridized modes may be expressed in terms of the following auxiliary quantities:

$$
\begin{aligned}
\epsilon_{1}= & -\left[\omega_{e}^{2}(\mathbf{q})+\omega_{e^{\prime}}^{2}(\mathbf{q})+\omega_{e^{\prime \prime}}^{2}(\mathbf{q})\right] \\
\epsilon_{2}= & \omega_{e}^{2}\left(\Delta_{0}^{2}+\tilde{\Delta}^{2}\right)+\omega_{e^{\prime}}^{2}\left(\Delta^{2}+\tilde{\Delta}^{2}\right)+\omega_{e^{\prime \prime}}^{2}\left(\Delta_{0}^{2}+\Delta^{2}\right) \\
& -\left(\Delta^{2} \Delta_{0}^{2}+\Delta^{2} \tilde{\Delta}^{2}+\Delta_{0}^{2} \tilde{\Delta}^{2}\right) \\
\epsilon_{3}= & 2 \Delta^{2} \Delta_{0}^{2} \tilde{\Delta}^{2}-\left(\omega_{e}^{2} \Delta_{0}^{2} \tilde{\Delta}^{2}+\omega_{e^{\prime}}^{2} \Delta^{2} \tilde{\Delta}^{2}+\omega_{e^{\prime \prime}}^{2} \Delta^{2} \Delta_{0}^{2}\right),
\end{aligned}
$$

with the definition of

$$
\begin{aligned}
& P=\epsilon_{2}-\frac{1}{3} \epsilon_{1}^{2} ; \quad Q=\frac{2}{27} \epsilon_{1}^{3}-\frac{1}{3} \epsilon_{1} \epsilon_{2}+\epsilon_{3}, \\
& \phi=\cos ^{-1}\left[-\frac{Q}{2}\left(\frac{|P|}{3}\right)^{-\frac{3}{2}}\right] .
\end{aligned}
$$

The dispersions of of the coupled modes $(i=1,2,3)$ are given by

$$
\omega_{i}^{2}(\mathbf{q})=2\left(\frac{|P|}{3}\right)^{\frac{1}{2}} \cos \left(\frac{\phi}{3}+\phi_{i}\right)-\frac{\epsilon_{1}}{3},
$$

where $\phi_{1}=0, \phi_{2}=\frac{2 \pi}{3}, \phi_{3}=\frac{4 \pi}{3}$. These expressions give the RPA mode dispersions for any splittings and matrix elements of the TSM and also for arbitrary temperatures. In terms of these modes, the collective RPA susceptibility may be written as

$$
\chi(\mathbf{q}, \omega)=\frac{\chi_{0}(\omega)}{\left[\omega^{2}-\omega_{1}^{2}(\mathbf{q})\right]\left[\omega^{2}-\omega_{2}^{2}(\mathbf{q})\right]\left[\omega^{2}-\omega_{3}^{2}(\mathbf{q})\right]} .
$$

This leads to a spectral function which determines the structure function in inelastic neutron scattering (INS),

$$
\frac{1}{\pi} \operatorname{Im} \chi(\mathbf{q}, \omega+i \eta)=\sum_{\lambda} R_{\lambda} \delta\left[\omega-\omega_{\lambda}(\mathbf{q})\right],
$$

with the momentum and temperature-dependent intensities $R_{\lambda}$ $(\lambda=1-3)$ of exciton modes given by

$$
\begin{aligned}
R_{\lambda}(\mathbf{q}, T) & =\frac{Z\left(\omega_{\lambda}(\mathbf{q})\right)}{2 \omega_{\lambda}(\mathbf{q}) \Pi_{\mu \neq \lambda}\left[\omega_{\mu}^{2}(\mathbf{q})-\omega_{\lambda}^{2}(\mathbf{q})\right]}, \\
Z\left(\omega_{\lambda}(\mathbf{q})\right) & =2\left[\alpha_{T}^{2} \Delta\left(\omega_{\lambda}(\mathbf{q})^{2}-\tilde{\Delta}^{2}\right)\left(\omega_{\lambda}(\mathbf{q})^{2}-\Delta_{0}^{2}\right)\right.
\end{aligned}
$$

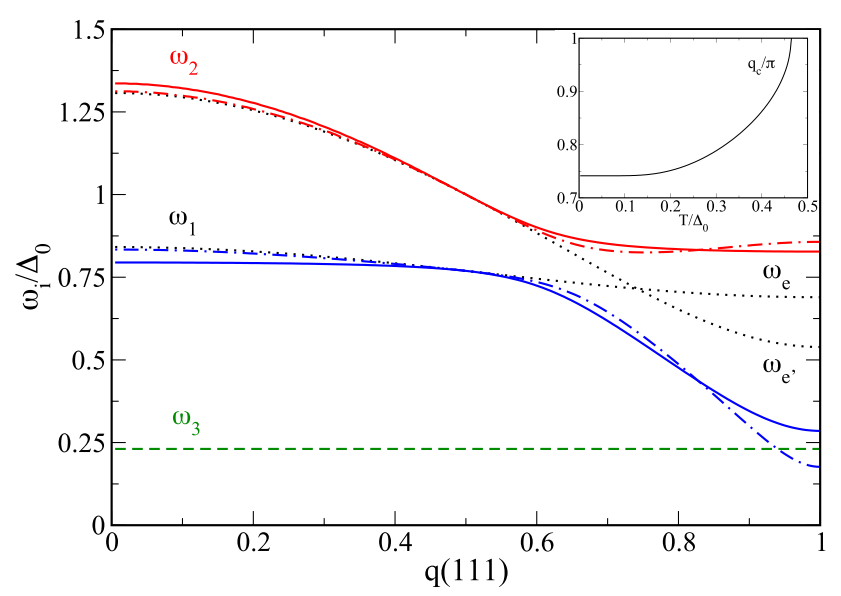

FIG. 3. Magnetic exciton dispersions along (111) direction, $q_{i}$ is in units of $\pi / a, \pi / b, \pi / c$ and $T / \Delta_{0}=0.1$. Dotted black lines: Uncoupled modes where the upper one has stronger dispersion due to $\beta>\alpha$. Full blue/red lines are the coupled RPA modes [Eqs. (20) and (16)]. The flat band (dashed green) corresponding to $1 \leftrightarrow 2$ excitations has vanishing intensity (Fig. 4). The hybridization gap is determined by the cross coupling $\sim \alpha \beta$ [Eq. (22)]. Dash-dotted blue/red lines show the dispersion for the main $\omega_{1,2}$ modes in Bogoliubov approach [Eq. (36)]. Inset: Crossing wave number $q_{c}$. Here $r=0.3, \xi_{\alpha}=0.2, \xi_{\beta}=0.71\left(\xi_{s}=0.91<1\right.$ subcritical $), \xi_{\tilde{\alpha}}=$ 0 [scheme as Fig. 2(a)]. 


$$
\begin{aligned}
& +\tilde{\alpha}_{T}^{2} \tilde{\Delta}\left(\omega_{\lambda}(\mathbf{q})^{2}-\Delta^{2}\right)\left(\omega_{\lambda}(\mathbf{q})^{2}-\Delta_{0}^{2}\right) \\
& \left.+\beta_{T}^{2} \Delta_{0}\left(\omega_{\lambda}(\mathbf{q})^{2}-\Delta^{2}\right)\left(\omega_{\lambda}(\mathbf{q})^{2}-\tilde{\Delta}^{2}\right)\right] .
\end{aligned}
$$

At low temperatures, we can find an approximate and more intuitive solution for the dispersions: For $T \ll \Delta, \Delta_{0}$ when $p_{12} \ll p_{01}, p_{02}$ we can neglect the second term in Eq. (6), i.e., the influence of transitions starting from the thermally excited states on the dynamics. Then the mode dispersions are obtained in concise form as

$$
\begin{aligned}
\omega_{1,2}^{2}(\mathbf{q})= & \frac{1}{2}\left(\omega_{e}^{2}+\omega_{e^{\prime}}^{2}\right) \pm\left[\frac{1}{4}\left(\omega_{e}^{2}-\omega_{e^{\prime}}^{2}\right)^{2}\right. \\
& \left.+\left(2 \alpha_{T} \beta_{T}\right)^{2}\left(I_{e} \Delta_{\mathrm{av}}\right)^{2}\right]^{\frac{1}{2}},
\end{aligned}
$$

with $\Delta_{\mathrm{av}}=\left(\Delta_{0}\right)^{\frac{1}{2}}$. The two dispersive modes stemming from the ground- to excited-state transitions may anticross if their dispersion is sufficiently strong, i.e., if $I_{0}$ intersite exchange is sufficiently large and matrix element $\alpha$ or $\beta$ large and sufficiently different. This happens when the decoupled dispersions fulfill $\omega_{e}\left(\mathbf{q}_{c}\right)=\omega_{e^{\prime}}\left(\mathbf{q}_{c}\right) \equiv \omega_{c}$. For $\mathbf{q}=(q, q, q)$ along $\Gamma R$ in the orthorhombic $\mathrm{BZ}$ where dispersion is maximal, one obtains

$$
q_{c}(T)=\cos ^{-1}\left(\frac{1}{12 I_{0}} \frac{\Delta_{0}^{2}-\Delta^{2}}{\beta_{T}^{2} \Delta_{0}-\alpha_{T}^{2} \Delta}\right)
$$

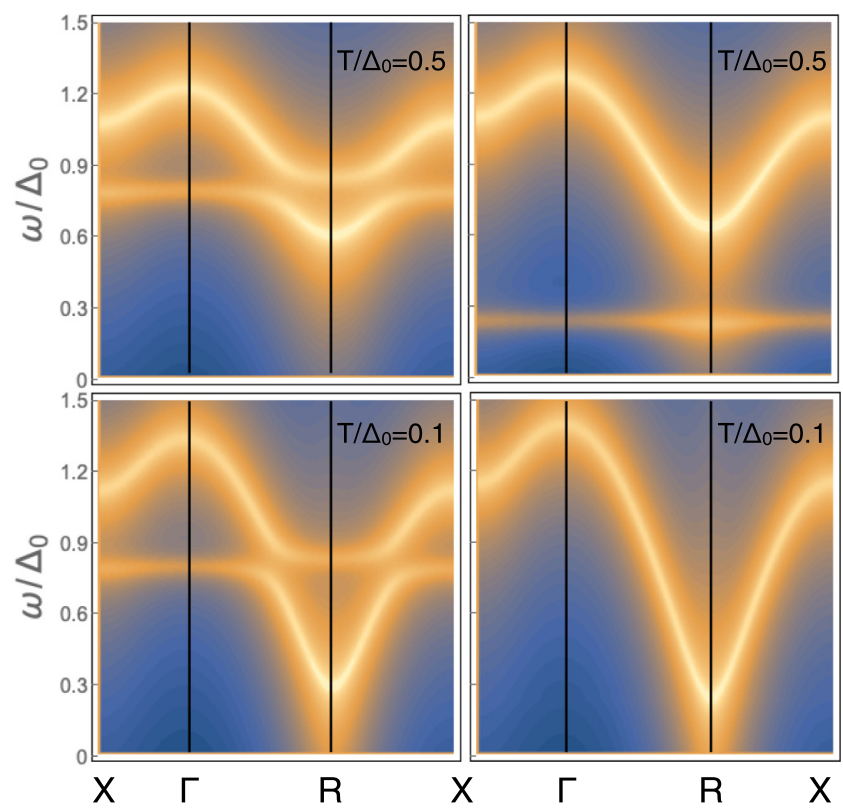

FIG. 4. Spectral density (log scale) of $\chi(\mathbf{q}, \omega)$ along scaled orthorhombic BZ path $\mathrm{X}(\pi, 0,0), \Gamma(0,0,0), \mathrm{R}(\pi, \pi, \pi), \mathrm{X}(\pi, 0,0)$ for two temperatures, using the residua given in Eqs. (19) and (23). Bright/dark colors correspond to large/small intensities. Left column: Scheme as Fig. 2(a), parameters as Fig. 3. Hybridizing modes $\omega_{1,2}$ with anticrossing are shown. For $T / \Delta_{0}=0.5$, dispersions are moderate, at $T / \Delta_{0}=0.1$ an incipient soft mode from $\omega_{1}(\mathbf{q})$ appears at the AF point (R) $\mathbf{Q}=(\pi, \pi, \pi)$ (cf. Fig. 3). Right column: Scheme as Fig. 2(b), parameters $r=0.3, \xi_{\beta}=0.94, \xi_{\tilde{\alpha}}=0.66, \xi_{\alpha}=$ 0 . For $T / \Delta_{0}=0.1$, a thermally excited flat mode $\left(\omega_{3}\right)$ appears. At $T / \Delta_{0}=0.1$, the latter is absent due to $p_{12} \ll 1$ and dispersion of $\omega_{2}(\mathbf{q})$ shows incipient soft mode behavior. A spectral broadening of $\eta=0.025$ is used. if the modulus of the argument is smaller than one. At the anticrossing point $q_{c}$ of the two exciton modes (Figs. 3 and 4), the splitting obtained from Eq. (20) is then given by

$$
\delta \omega_{c}=\omega_{1}\left(q_{c}\right)-\omega_{2}\left(q_{c}\right)=2\left|\alpha_{T} \beta_{T}\right|\left|I_{e}\left(q_{c}\right)\right| \frac{\Delta_{\mathrm{av}}}{\omega_{c}} .
$$

The anticrossing happens because both inelastic transitions start from the same ground state and the splitting is therefore $\sim\left|\alpha_{T} \beta_{T}\right|$. The dispersion as well as the splitting decrease with increasing $T$ due to the reduction of effective transition strengths $\alpha_{T} \sim p_{01}$ and $\beta_{T} \sim p_{02}$ (Fig. 1). The intensities determining the spectral functions now take on the simplified form

$$
\begin{aligned}
R_{\lambda}(\mathbf{q}, T) & =\frac{Z\left(\omega_{\lambda}\right)}{2 \omega_{\lambda}(\mathbf{q})\left[\omega_{\bar{\lambda}}^{2}(\mathbf{q})-\omega_{\lambda}^{2}(\mathbf{q})\right]}, \\
Z\left(\omega_{\lambda}\right) & =2\left[\alpha_{T}^{2} \Delta\left(\omega_{\lambda}(\mathbf{q})^{2}-\Delta_{0}^{2}\right)+\beta_{T}^{2} \Delta_{0}\left(\omega_{\lambda}(\mathbf{q})^{2}-\Delta^{2}\right)\right]
\end{aligned}
$$

where $\bar{\lambda}=2,1$ for $\lambda=1,2$, respectively. A discussion of exciton mode dispersions and intensities is given at the end of Sec. IV.

\section{DIAGONALIZATION BY BOGOLIUBOV TRANSFORMATION AND EXCITONIC BLOCH STATES}

The response function formalism leads to a transparent picture for the excitonic mode dispersions, however, it gives no information on the Bloch functions of these modes. For that purpose, a direct (approximate) diagonalization of the Hamiltonian using pseudounitary Bogoliubov and subsequent unitary transformations may be performed that also contain the eigenvectors of exciton modes. Therefore, we also apply this alternative approach to the problem. In this context, the local CEF excitation standard basis operators $|i\rangle\langle j|$ in the TSM are mapped to bosons (although the former have more complicated commutation relations). This can be justified as long as the temperature fulfills $T \ll \Delta, \Delta_{0}$ and only the two excitations from the ground state have to be considered [27,31], corresponding to the TSM of Fig. 2(a). Defining $a_{i}^{\dagger}=|1\rangle\langle 0|$ and $b_{i}^{\dagger}=|2\rangle\langle 0|$ and using the Fourier transforms $x_{\mathbf{k}}^{\dagger}=(1 / \sqrt{N}) \sum_{i} \exp \left(-i \mathbf{k} \mathbf{R}_{i}\right) x_{i}^{\dagger}(x=a, b)$, the Hamiltonian $H=H_{\mathrm{CEF}}+H_{\mathrm{ex}}$ may be written in its bosonic form by using the definition $\psi_{\mathbf{k}}^{\dagger}=\left(a_{\mathbf{k}}^{\dagger}, a_{-\mathbf{k}}, b_{\mathbf{k}}^{\dagger}, b_{-\mathbf{k}}\right)$ as $H=\sum_{\mathbf{k}} \psi_{\mathbf{k}}^{\dagger} h_{\mathbf{k}} \Psi_{\mathbf{k}}+$ $\frac{1}{2} N\left(\Delta+\Delta_{0}\right)$, where (k suppressed on right side)

$$
h_{\mathbf{k}}=\frac{1}{2}\left(\begin{array}{cccc}
\Omega_{a} & -\alpha^{2} I_{e} & -\alpha \beta I_{e} & -\alpha \beta I_{e} \\
-\alpha^{2} I_{e} & \Omega_{a} & -\alpha \beta I_{e} & -\alpha \beta I_{e} \\
-\alpha \beta I_{e} & -\alpha \beta I_{e} & \Omega_{b} & -\beta^{2} I_{e} \\
-\alpha \beta I_{e} & -\alpha \beta I_{e} & -\beta^{2} I_{e} & \Omega_{b}
\end{array}\right) .
$$

Here we defined

$$
\Omega_{\mathbf{k}}^{a}=\Delta-\alpha^{2} I_{e}^{\mathbf{k}}, \quad \Omega_{\mathbf{k}}^{b}=\Delta_{0}-\beta^{2} I_{e}^{\mathbf{k}} .
$$

This Hamiltionian may be approximately diagonalized by pseudounitary Bogoliubov transformations in each particlehole subspace of $a, b$-type operators and a subsequent unitary rotation in the space of isolated $A, B$ normal modes. The 
former are given by

$$
\begin{aligned}
& A_{\mathbf{k}}=\cosh \theta_{\mathbf{k}}^{a} a_{\mathbf{k}}+\sinh \theta_{\mathbf{k}}^{a} a_{-\mathbf{k}}^{\dagger}, \\
& B_{\mathbf{k}}=\cosh \theta_{\mathbf{k}}^{b} b_{\mathbf{k}}+\sinh \theta_{\mathbf{k}}^{b} b_{-\mathbf{k}}^{\dagger},
\end{aligned}
$$

which preserve the bosonic commutation relations for the $A_{\mathbf{k}}, B_{\mathbf{k}}$. The above transformation diagonalizes each diagonal $2 \times 2$ block in Eq. (24) when the conditions

$$
\begin{aligned}
& \tanh 2 \theta_{\mathbf{k}}^{a}=-\frac{\alpha^{2} I_{e}^{\mathbf{k}}}{\Omega_{\mathbf{k}}^{a}}=\frac{\Omega_{\mathbf{k}}^{a}-\Delta}{\Omega_{\mathbf{k}}^{a}}, \\
& \tanh 2 \theta_{\mathbf{k}}^{b}=-\frac{\beta^{2} I_{e}^{\mathbf{k}}}{\Omega_{\mathbf{k}}^{b}}=\frac{\Omega_{\mathbf{k}}^{b}-\Delta_{0}}{\Omega_{\mathbf{k}}^{b}}
\end{aligned}
$$

are fulfilled. This leads to the transformed Hamiltionian (in $A, B$ particle space only) in terms of $A, B$ uncoupled normal mode coordinates given by

$$
\begin{aligned}
& H=E_{0}+\sum_{\mathbf{k}}\left(A_{\mathbf{k}}^{\dagger}, B_{\mathbf{k}}^{\dagger}\right)\left(\begin{array}{cc}
\omega_{\mathbf{k}}^{A} & 2 \tilde{\lambda}_{\mathbf{k}} \\
2 \tilde{\lambda}_{\mathbf{k}} & \omega_{\mathbf{k}}^{B}
\end{array}\right)\left(\begin{array}{l}
A_{\mathbf{k}} \\
B_{\mathbf{k}}
\end{array}\right), \\
& E_{0}=\frac{1}{2} \sum_{\mathbf{k}}\left[\left(\omega_{\mathbf{k}}^{A}-\Delta\right)+\left(\omega_{\mathbf{k}}^{B}-\Delta_{0}\right)\right] .
\end{aligned}
$$

Here $\omega_{\mathbf{k}}^{A}$ and $\omega_{\mathbf{k}}^{B}$ are the uncoupled normal mode frequencies,

$$
\omega_{\mathbf{k}}^{A}=\left[\Delta\left(\Delta-2 \alpha^{2} I_{e}^{\mathbf{k}}\right)\right]^{\frac{1}{2}} ; \quad \omega_{\mathbf{k}}^{B}=\left[\Delta_{0}\left(\Delta_{0}-2 \beta^{2} I_{e}^{\mathbf{k}}\right)\right]^{\frac{1}{2}},
$$

which are indeed equivalent to the uncoupled exciton modes $\omega_{e}, \omega_{e^{\prime}}$, respectively, of the RPA response function approach in Eqs. (12) for the low-temperature limit. Furthermore, they satisfy the relations

$$
\Omega_{\mathbf{k}}^{a 2}-\omega_{\mathbf{k}}^{A 2}=\left(\Omega_{\mathbf{k}}^{a}-\Delta\right)^{2}, \quad \Omega_{\mathbf{k}}^{b 2}-\omega_{\mathbf{k}}^{B 2}=\left(\Omega_{\mathbf{k}}^{b}-\Delta_{0}\right)^{2} .
$$

The coupling term in Eqs. (28) obtained through the transformation described by Eq. (26) is given by

$$
\tilde{\lambda}_{\mathbf{k}}=-\frac{1}{2}(\alpha \beta) I_{e}^{\mathbf{k}}\left(u_{\mathbf{k}}^{a}-v_{\mathbf{k}}^{a}\right)\left(u_{\mathbf{k}}^{b}-u_{\mathbf{k}}^{b}\right),
$$

with $u_{\mathbf{k}}^{a, b}=\cosh \theta_{\mathbf{k}}^{a, b}, v_{\mathbf{k}}^{a, b}=\sinh \theta_{\mathbf{k}}^{a, b}$. It may be evaluated using Eq. (27) as

$$
\tilde{\lambda}_{\mathbf{k}}=-\frac{1}{2} \alpha \beta I_{e}^{\mathbf{k}} \frac{\Delta_{\mathrm{av}}^{2}}{\omega_{\mathbf{k}}^{A} \omega_{\mathbf{k}}^{B}} .
$$

Now a further unitary transformation in $A, B$ particle space can be employed according to

$$
\begin{aligned}
& \chi_{1 \mathbf{k}}=\cos \phi_{\mathbf{k}} A_{\mathbf{k}}+\sin \phi_{\mathbf{k}} B_{\mathbf{k}}, \\
& \chi_{2 \mathbf{k}}=-\sin \phi_{\mathbf{k}} A_{\mathbf{k}}+\cos \phi_{\mathbf{k}} B_{\mathbf{k}} .
\end{aligned}
$$

These are the normal mode exciton coordinates that diagonalize the Hamiltonian in Eq. (24) (up to residual two-exciton interactions), provided the condition

$$
\tan 2 \phi_{\mathbf{k}}=\frac{4 \tilde{\lambda}_{\mathbf{k}}}{\omega_{\mathbf{k}}^{A}-\omega_{\mathbf{k}}^{B}}= \pm\left[\left(\frac{\omega_{1 \mathbf{k}}-\omega_{2 \mathbf{k}}}{\omega_{\mathbf{k}}^{A}-\omega_{\mathbf{k}}^{B}}\right)^{2}-1\right]^{\frac{1}{2}}
$$

is fulfilled, leading to

$$
H=E_{0}+\sum_{\mathbf{k}}\left[\omega_{1 \mathbf{k}} \chi_{1 \mathbf{k}}^{\dagger} \chi_{1 \mathbf{k}}+\omega_{2 \mathbf{k}} \chi_{2 \mathbf{k}}^{\dagger} \chi_{2 \mathbf{k}}\right]
$$

where the exciton mode frequencies are finally given by

$$
\omega_{(1,2) \mathbf{k}}=\frac{1}{2}\left(\omega_{\mathbf{k}}^{A}+\omega_{\mathbf{k}}^{B}\right) \pm\left[\frac{1}{4}\left(\omega_{\mathbf{k}}^{A}-\omega_{\mathbf{k}}^{B}\right)^{2}+4 \tilde{\lambda}_{\mathbf{k}}^{2}\right]^{\frac{1}{2}},
$$

which essentially corresponds to the RPA result of Eq. (20) for zero temperature. Obviously, the direct diagonalization route to obtain the exciton modes is more elaborative than the response function formalism. On the other hand, it also provides the Bloch functions $\chi_{1,2 \mathbf{k}}^{\dagger}|0\rangle$ whose creation operators are, according to Eqs. (26) and (33), explicitly given by

$$
\begin{aligned}
& \chi_{1 \mathbf{k}}^{\dagger}=c \tilde{c}_{a} a_{\mathbf{k}}^{\dagger}+c \tilde{s}_{a} a_{-\mathbf{k}}+s \tilde{c}_{b} b_{\mathbf{k}}^{\dagger}+s \tilde{s}_{b} b_{-\mathbf{k}}, \\
& \chi_{2 \mathbf{k}}^{\dagger}=-s \tilde{c}_{a} a_{\mathbf{k}}^{\dagger}-s \tilde{s}_{a} a_{-\mathbf{k}}+c \tilde{c}_{b} b_{\mathbf{k}}^{\dagger}+c \tilde{s}_{b} b_{-\mathbf{k}},
\end{aligned}
$$

where we defined $s=\sin \phi_{\mathbf{k}}, c=\cos \phi_{\mathbf{k}}$ and $\tilde{s}_{a, b}=$ $\sinh \theta_{\mathbf{k}}^{a, b}, \tilde{c}_{a, b}=\cosh \theta_{\mathbf{k}}^{a, b}$. They fulfill the standard bosonic commutation relations $\left[\chi_{n \mathbf{k}}, \chi_{n^{\prime} \mathbf{k}^{\prime}}^{\dagger}\right]=\delta_{n n^{\prime}} \delta_{\mathbf{k k}^{\prime}}(n=1,2)$.

We can give explicit expressions for the transformation coefficients in Eq. (37) in terms of the various isolated and coupled eigenmode frequencies by eliminating the angles $\theta_{\mathbf{k}}^{a, b}$ and $\phi_{\mathbf{k}}$. Using Eqs. (27) and (34), we obtain

$$
\tilde{c}_{a, b}=\left[\frac{1}{2}\left(\frac{\Omega_{\mathbf{k}}^{a, b}}{\omega_{\mathbf{k}}^{A, B}}+1\right)\right]^{\frac{1}{2}}, \quad \tilde{s}_{a, b}=\left[\frac{1}{2}\left(\frac{\Omega_{\mathbf{k}}^{a, b}}{\omega_{\mathbf{k}}^{A, B}}-1\right)\right]^{\frac{1}{2}}
$$

for the Bogoliubov transformation coefficients. Likewise, we get

$$
\begin{aligned}
& c=\left[\frac{1}{2}\left(1+\frac{\left|\omega_{\mathbf{k}}^{A}-\omega_{\mathbf{k}}^{B}\right|}{\left|\omega_{1 \mathbf{k}}-\omega_{2 \mathbf{k}}\right|}\right)\right]^{\frac{1}{2}}, \\
& s=\left[\frac{1}{2}\left(1-\frac{\left|\omega_{\mathbf{k}}^{A}-\omega_{\mathbf{k}}^{B}\right|}{\left|\omega_{1 \mathbf{k}}-\omega_{2 \mathbf{k}}\right|}\right)\right]^{\frac{1}{2}}
\end{aligned}
$$

for the coefficients of the subsequent unitary transformation.

The comparison of the low excitonic modes at low temperature as obtained from response function and Bogoliubov approach is shown in Fig. 3 for the (111) direction. Control parameters are chosen such that a crossing of uncoupled modes (dotted lines) of Eqs. (12) and (29) occurs at wave number $q_{c}$. Their hybridization leads to an anticrossing of the coupled modes [Eqs. (16), (20), and (36)]. The full line represents the RPA result of Eqs. (16) and (20). The inset depicts the increase of the crossing wave number with temperature. Once it has reached the zone boundary, the modes become gradually decoupled due the suppression of their dispersion. At the AF zone boundary vector $\mathbf{Q}(q / \pi=1)$, the lower mode $\omega_{1}(\mathbf{q})$ shows incipient softening. The dash-dotted line is obtained from the Bogoliubov result in Eq. (36) and is rather close to the full line. There are, however, distinct differences close to the AF point: Because the effective hybridization $\tilde{\lambda}_{\mathbf{k}}$ [Eq. (32)] is enhanced by a feedback effect due to the mode softening, the latter happens more rapidly in the Bogoliubov approach. This will also lead to a difference in the phase boundary for the two techniques (inset of Fig. 5).

The temperature dependence of spectral functions for TSMs of Figs. 2(a) and 2(b) obtained from RPA theory [Eqs. (19)] is presented in Fig. 4 (left and right columns, respectively) and shows distinctive features. Left: (i) With increasing temperature, the anticrossing region moves to larger wave vectors, concomitant with $q_{c}(T)$ in Fig. 3. (ii) With decreasing temperature, $\omega_{1}(\mathbf{q})$ becomes an incipient soft mode. 


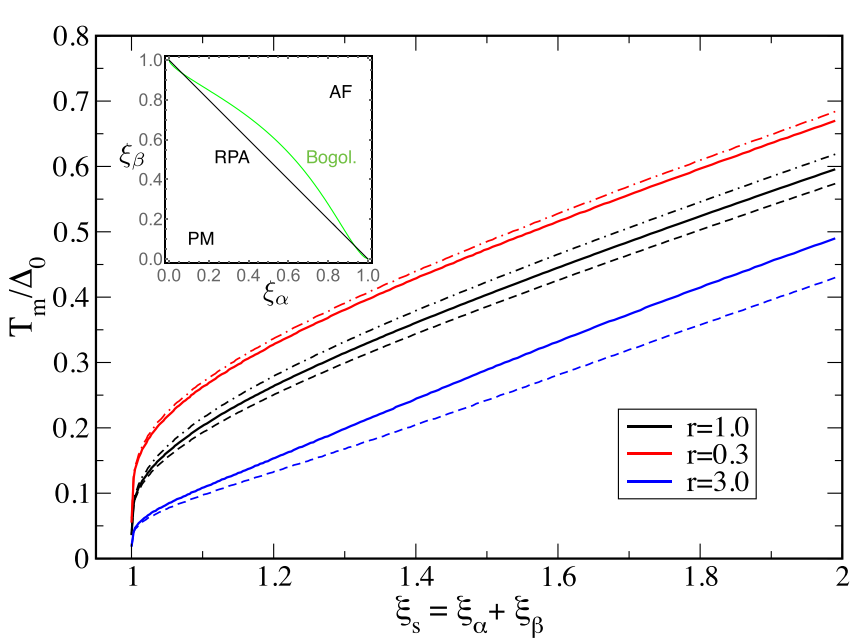

FIG. 5. The critical temperature for induced TSM order as function of control parameters $\xi_{s}$ for $\xi_{a}<1$ and splitting ratio $r=\tilde{\Delta} / \Delta$. Here full lines correspond to $\xi_{a}=0$, dashed/dashed-dotted lines to $\xi_{a}=-0.2,0.2$ (black), and $\xi_{a}=-0.3,0.3$ (blue and red, respectively). Inset: PM/AF phase boundary $T_{m}\left(\xi_{\alpha}, \xi_{\beta}\right)=0$ for symmetric case $r=1$ from RPA (black) and Bogoliubov (green) theories.

For slightly larger $\xi_{\beta}$, it would become unstable at lowest temperature. Correspondingly, the dispersive width of $\omega_{2}(\mathbf{q})$ increases for lower temperatures. Right: (i) At larger temperatures, the mostly flat $\omega_{3}(\mathbf{q})$ low-energy mode originating from transitions between thermally excited $|1\rangle$ and $|2\rangle$ states is still visible, its flatness is caused by the always small thermal population difference factor $p_{12}$ (Fig. 1). For this reason, its spectral weight also decays exponentially at low temperature and therefore it has vanished from Fig. $4\left(T / \Delta_{0}=0.1\right)$. (ii) The $\omega_{2}(\mathbf{q})$ mode now shows incipient soft mode behavior due to slightly below-critical control parameters.

\section{SOFT MODE BEHAVIOR AND CRITICAL CONDITION FOR MAGNETIC ORDER}

When temperature is lowered, the effective coupling parameters $\alpha_{T}^{2}=\alpha^{2} p_{01}, \beta_{T}^{2}=\beta^{2} p_{02}$ for the TSM of Fig. 2(a) increase and with it the dispersive width of $\omega_{1}, \omega_{2}$ modes. Eventually, one of them may touch zero at the wave vector $\mathbf{q}$ where $I_{e}(\mathbf{q})$ has its maximum, frequently (but not necessarily) at zone center $\mathbf{q}=0$ or boundary $\mathbf{Q}=(\pi, \pi, \pi)$. This mode softening signifies the onset of induced excitonic FM, AF quantum magnetism at $T_{m}$, respectively. In distinction to common magnetic order, the moments are not preformed already at larger temperatures and order at $T_{m}$, since there are only nonmagnetic singlet states available, but rather the creation and ordering of moments happens simultaneously at $T_{m}$ due to off-diagonal virtual transitions between the singlets.

We first consider the soft mode condition within the RPA response function formalism. According to Eq. (17), it is equivalent to the divergence of the static susceptibility $\chi\left(\mathbf{q}, T_{m}\right)^{-1} \rightarrow 0$ which leads to the criticality condition

$$
\chi_{0}\left(0, T_{m}\right)=\frac{1}{I_{e}(\mathbf{q})}
$$

This means that the static $\left(i \omega_{n}=0\right)$ single-ion susceptibility given by Eq. (6) must reach a mininum value $\geqslant 1 / I_{e}(\mathbf{q})$ to achieve induced magnetic order at finite $T_{m}$. We focus on the AF case $\left(I_{0}<0\right)$ where this is first fulfilled for the AF wave vector $\mathbf{q}=\mathbf{Q}$. The procedure for $\mathrm{FM}(\mathbf{q}=0)$ or even incommensurate cases are analogous.

As a reference, we recapitulate the well-known expression for $T_{m}$ in the $t w o$-singlet model [5,7,14,28] (e.g., taking off the upper singlet-state $|2\rangle$ in Fig. 1). In this case, $\left(I_{e} \equiv I_{e}(\mathbf{Q})=\right.$ $\left.-z I_{0}>0\right)$ :

$$
T_{m}=\frac{\Delta}{2 \tanh ^{-1} \frac{1}{\xi_{\alpha}}}, \quad \xi_{\alpha}=\frac{2 \alpha^{2} I_{e}}{\Delta},
$$

where $\xi_{\alpha}$ is now the only dimensionless control parameter of the model and at $\xi_{\alpha}^{c}=1$ a quantum phase transition from paramagnetic $\xi<\xi_{\alpha}^{c}$ to magnetic $\xi>\xi_{\alpha}^{c}$ ground state appears. In the marginally critical case $\xi_{\alpha}=1+\delta(0<\delta \ll 1)$, we can expand $T_{m} \simeq \Delta / \ln \left(\frac{2}{\delta}\right)$ and thus the ordering temperature vanishes logarithmically when approaching the critical value $\xi_{\beta}^{c}(\delta \rightarrow 0)$ from above. This is a characteristic behavior of an induced excitonic quantum magnet.

Now we consider the extended TSM cases of Fig. 2 with generally possible parameter sets. The critical equation for $T_{m}$ [Eqs. (41)] may be written with the use of control parameters of Eqs. (2) as

$$
\xi_{\alpha} p_{01}\left(T_{m}\right)+\xi_{\beta} p_{02}\left(T_{m}\right)+\xi_{\tilde{\alpha}} p_{12}\left(T_{m}\right)=1 .
$$

For convenience, we now define the splitting ratio $r=\tilde{\Delta} / \Delta$, meaning $\Delta=\Delta_{0} /(1+r)$. Then $r=1$ corresponds to the symmetric case with $\Delta=\tilde{\Delta}$ (Fig. 1) and $r \neq 1$ to the general asymmetric case. Defining furthermore $y=\exp (\Delta / T)$, the critical condition for induced order Eq. (40) can be written as

$$
\left(1-\xi_{s}\right) y_{m}^{r+1}+\left(1-\xi_{a}\right) y_{m}^{r}+\left(1+\xi_{s}+\xi_{a}\right)=0,
$$

then $T_{m}=\Delta / \ln y_{m}$ is the ordering temperature with $y_{m}$ given by the solution of the above algebraic equation. Note that even in the general asymmetric TSM described by Eq. (43), there are effectively two control parameters which are combinations of the three possible parameters in Eqs. (2) according to $\xi_{s}=\xi_{\alpha}+\xi_{\beta}$ and $\xi_{a}=\xi_{\tilde{\alpha}}-\xi_{\alpha}$, leading explicitly to the expressions

$$
\xi_{s}=\frac{2 I_{e}}{\Delta}\left(\frac{\beta^{2}}{1+r}+\alpha^{2}\right), \quad \xi_{a}=\frac{2 I_{e}}{\Delta}\left(\frac{\tilde{\alpha}^{2}}{r}-\alpha^{2}\right) .
$$

We should remember that in the paramagnetic state, one of the elements in the se $(\alpha, \beta, \tilde{\alpha})$ must be zero, corresponding to the cases of Fig. 2. The solution of Eq. (43) for finite $T_{m}$ and general splitting ratio $r$ is only possible numerically. However, for discrete values like $r=\frac{1}{2}, 1,2,3$, explicit solutions for $T_{m}$ can be obtained but except for $r=1$ are not particularly instructive. We may also look at the limiting cases $r \rightarrow 0, \infty$. The latter corresponds to the singlet-singlet model and recovers the solution in Eqs. (41) while the former describes the singlet-doublet model [11] with splitting $\Delta$. Its $T_{m}$ is also described by Eqs. (41) but with the replacement $\xi_{\beta} \rightarrow 2\left(\alpha^{2}+\beta^{2}\right) I_{e} / \Delta$.

Now we discuss two typical special cases of the TSM model where the solution for $T_{m}$ can be obtained in closed 


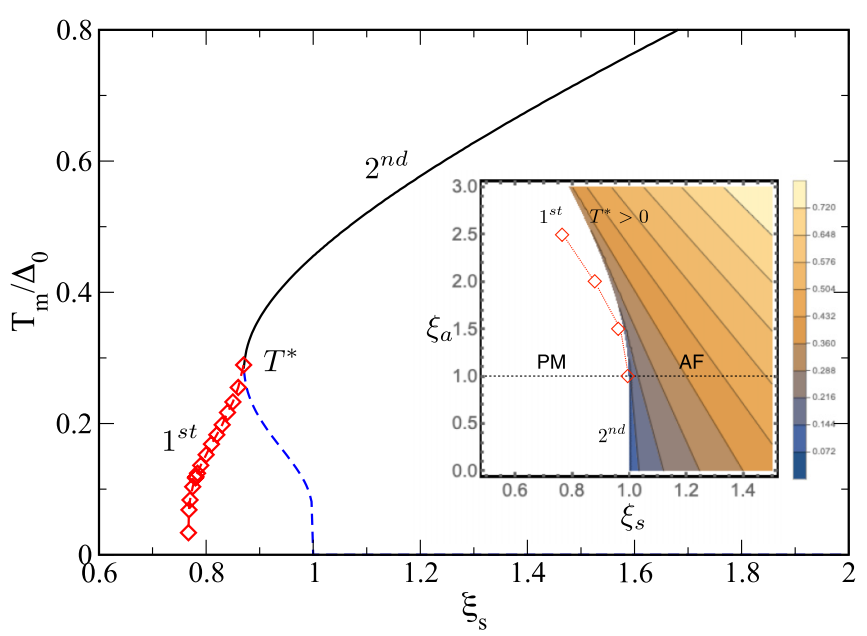

FIG. 6. Critical temperature as function of $\xi_{s}$ for $\xi_{a}=2.5>1$ for the weakly symmetric case $(r=1)$. Here full black line corresponds to second-order transition obtained from physical solution of Eq. (43) as in Figs. 5 and 7 and red symbols and dashed line denote a first-order transition (cf. Fig. 8). Here $T^{*}$ denotes the critical point. The dashed blue line gives the lower unphysical solution of Eq. (43). Inset: Phase diagram of induced AF order (weakly symmetric $r=1$ TSM) in the control parameter plane $\left(\xi_{s}, \xi_{a}\right)$. For $\xi_{a}<1$, the $T_{m}=0$ phase boundary does not depend on $\xi_{a}$ (cf. Fig. 5 and Sec. V). For $\xi_{a}>1, T^{*}$ maps the line of critical points between first- and secondorder regimes and the red symbols trace the first-order transition line $T_{m}=0$. The blue lower corner corresponds to the inverse logarithmic decrease of $T_{m} / \Delta_{0}$ evident from Fig. 5.

form from Eq. (43). These are considerably more complicated to derive than for the two-level system but formally similar:

(i) Weakly symmetric TSM $r=1$ but $\tilde{\alpha} \neq \alpha$.

Then, Eq. (43) reduces to a quadratic equation and from its two solutions $y_{m}^{ \pm}$the critical temperature may be obtained as

$$
T_{m}^{ \pm}=\frac{\Delta}{2 \tanh ^{-1} \frac{1}{\eta_{s}^{ \pm}}}, \quad \frac{1}{\eta_{s}^{ \pm}}=\frac{y_{m}^{ \pm}-1}{y_{m}^{ \pm}+1} .
$$

Explicitly, one obtains after some derivations,

$$
\frac{1}{\eta_{s}^{ \pm}}=\frac{2 \xi_{s}+\xi_{a} \pm\left[\left(4 \xi_{s}^{2}-3\right)+2 \xi_{a}\left(2 \xi_{s}-3\right)+\xi_{a}^{2}\right]^{\frac{1}{2}}}{1+2 \xi_{a}}
$$

where $\xi_{s}, \xi_{a}$ are given by Eqs. (44) with $r=1$. Instead of directly having the control parameter $\xi_{s}$ appearing in $T_{m}$ as in Eqs. (41), it is replaced by a function $\eta_{s}\left(\xi_{s}, \xi_{a}\right)$. A solution for finite $T_{m}^{ \pm}$exists only when $\eta_{s}^{ \pm}\left(\xi_{s}, \xi_{a}\right) \geqslant 1$. The physical solution is always $T_{m} \equiv T_{m}^{-}$with $\eta_{s}=\eta_{s}^{-}$. The second solution $T_{m}^{+}$does not exist for $\xi_{a}<1$ and for $\xi_{a}>1$ corresponds to the unphysical branch with $T_{m}^{-}<T_{m}$ (blue dashed line in Fig. 6). It is easy to show that $\eta_{s}^{-1}\left(1, \xi_{a}\right)=1$ for $\xi_{a} \leqslant 1$. Therefore, the $T_{m}$ and the phase boundary position $\xi_{s}$ does not depend on $\xi_{a}$ in this case as is indeed demonstrated by Fig. 5 and inset of Fig. 6.

(ii) Fully symmetric TSM $r=1$ and $\tilde{\alpha}=\alpha$.

This means that now $\xi_{a}=0$ and only one effective control parameter $\xi_{s}$ remains. $T_{m}$ is given by the same expression as above but with the simplification

$$
\begin{aligned}
\eta_{s}^{-1} & =\left[2 \xi_{s}-\left[4 \xi_{s}^{2}-3\right]^{\frac{1}{2}}\right], \\
\xi_{s} & =\frac{2\left(\alpha^{2}+\frac{1}{2} \beta^{2}\right) I_{e}}{\Delta},
\end{aligned}
$$

where now $\xi_{s}$ is the control parameter for the fully symmetric TSM that contains both matrix elements and the splitting $\Delta=\Delta_{0} / 2$. For a finite $T_{m}$, one must have $\eta_{s}>1$ and hence $\xi_{s}>1$. In the marginal critical case $\xi_{s}=1+\delta$ the transition temperature shows similar logarithmic behavior as before, but with $T_{m} \simeq \Delta / \ln \left(\frac{1}{\delta}\right)$.

The systematic variation of $T_{m}\left(\xi_{s}, \xi_{a} ; r\right)$ is shown in Fig. 5 for $\xi_{a}<1$. The fully and weakly symmetric cases $(r=1)$ discussed in detail above correspond to the full and broken black lines in Fig. 5, respectively. In the asymmetric case $(r \neq 1)$, the transition temperature $T_{m}$ changes considerably with the splitting asymmetry $r=\tilde{\Delta} / \Delta$, keeping the total splitting $\Delta_{0}$ constant. When $r<1$, the central state $|1\rangle$ is shifted upward, leading to an increased effectiveness because the occupation difference $p_{01}$ increases, therefore $T_{m}$ increases. The reversed argument holds for $r>1$. Furthermore, when the asymmetric control parameter $\xi_{a}$ is larger or smaller than zero for a given $r$, the value of $T_{m}$ moderately increases or decreases, respectively.

For $\xi_{a}>1$, when the coupling of thermally excited states becomes important, a surprising new situation occurs (Fig. 6): First, the second-order transition temperature $T_{m}$ now stays finite for $\xi_{s}<1$ and, second, at a certain critical point $T_{m}=T^{*}$ it changes into a first-order transition for $T_{m}<T^{*}$. This is, of course, no longer described by Eq. (43) and its special cases since it was obtained from the divergence of the susceptibility at $T_{m}$. Below $T^{*}$, this is no longer true and $T_{m}$ has to be determined by solving directly the self-consistency equations for the order parameter (Sec. VI). The resulting line of first-order transitions is shown by red symbols and dashed line in the main Fig. 6 for $\xi_{a}=2.5$. For this value, the first-order line stops at $\xi_{s}=0.77$.

Alternatively, this variation can be combined in a contour plot of $T_{m}$ in the $\left(\xi_{s}, \xi_{a}\right)$ control parameter plane for fixed $r$, taken as the symmetric case $r=1$ in the inset of Fig. 6. First, it shows that the sector of first-order transitions bounded by the red symbols and broken line to the left and the $T^{*}>0$ line to the right widens when $\xi_{a}$ increases, i.e., the transitions between thermally excited states become more important. Second, it shows explicitly the $\xi_{s}$ independence of the second order PM/AF phase boundary defined by $T_{m}\left(\xi_{s}, \xi_{a} ; r\right)=0$ for $\xi_{a}<1$ as already noticed before. This property may be traced back directly to the fundamental equation for $T_{m}$ given by Eq. (43).

In this respect, it is instructive to compare the predictions of the soft mode conditions $\omega_{2}(\mathbf{Q})=0$ at $T_{m}=0$ for RPA [Eq. (20)] and Bogoliubov [Eq. (36)] approaches for consistency [in the case $\tilde{\alpha}=0$ of Fig. 2(a)]. They cannot be identical due to the slightly different expressions for the exciton mode dispersions. In the RPA case, one simply obtains from the equivalent Eq. (43) in the limit $y_{m} \rightarrow \infty: \xi_{s}=\xi_{\alpha}+\xi_{\beta}=1$, in accordance with the previous discussion of symmetric models (inset of Fig. 6 for $\xi_{a}<1$ ). This means the effect of the two excitations is simply additive at the phase boundary. In 
comparison, the Bogoliubov case leads to the more complicated relation:

$$
\left[\left(\xi_{\alpha}-1\right)\left(\xi_{\beta}-1\right)\right]=\left(\frac{1}{4} \xi_{\alpha} \xi_{\beta}\right)^{\frac{1}{3}} .
$$

For the special case $\xi_{\alpha}=\xi_{\beta}=\xi$, we obtain $\xi=0.5$ in the RPA approach and $\xi=0.57$ in the Bogoliubov approach. Furthermore, in both cases the boundary points $\left(\xi_{\alpha}, \xi_{\beta}\right)=$ $(1,0),(0,1)$ are identical for both methods. The complete comparison of $\mathrm{PM} / \mathrm{AF}$ phase boundaries $T_{m}\left(\xi_{\alpha}, \xi_{\beta}\right)=0$ is shown in the inset of Fig. 5 for both methods. It demonstrates a rather close agreement between the two technically rather different approaches.

\section{THE INDUCED ORDER PHASE AND ITS EXCITATIONS}

We now consider the phase with induced magnetic order in the TSM. To be specific, we treat only the AF case corresponding to the soft mode with at $\mathbf{Q}=(\pi, \pi, \pi)$. The more direct treatment is based on the RPA approach with the inclusion of the mean field induced order. The alternative would be the exciton condensation picture for Bogoliubov quasiparticles. The latter is problematic to extrapolate to the disordered phase with temperatures considerably above $T_{m}$ due to the influence of thermally populated CEF singlets. This is no problem for the response function approach which will therefore be used here. As a necessary basis, we need the mean-field self-consistency equation for the induced order parameter. The CEF molecular field Hamiltonian is given by

$$
\begin{aligned}
& H_{\mathrm{CEF}}^{\mathrm{mf}}=H_{\mathrm{CEF}}-\sum_{l} h_{e}(l) J_{z}(l), \\
& h_{e}(l)=\sum_{l^{\prime}} I_{l l^{\prime}}\left\langle J_{z}\left(l^{\prime}\right)\right\rangle,
\end{aligned}
$$

with the exchange model of Eq. (10) the effective molecular field on the two AF sublattices $A, B$ is $h_{e}^{A, B}=I_{e}\left\langle J_{z}^{B, A}\right\rangle$ where $I_{e}=z\left|I_{0}\right|\left(I_{0}<0\right.$ for AF exchange $)$ and $\left\langle J_{z}^{A, B}\right\rangle= \pm\left\langle J_{z}\right\rangle$. The associated difference in free energy per site between induced moment state and paramagnetic state corresponding to Eqs. $(48,1)$ is given by

$$
\delta F / N=-I_{e}\left\langle J_{z}\right\rangle^{2}-T \sum_{i}\left(p_{i}^{\prime} \ln p_{i}^{\prime}-p_{i} \ln p_{i}\right)
$$

where the $p_{i}$ are the paramagnetic CEF level occupations (Sec. II) and $p_{i}^{\prime}$ the occupation of levels in the $\mathrm{AF}$ state, renormalized by the molecular field. Explicitly, $p_{i}^{\prime}=$ $Z^{\prime-1} \exp \left(-E_{i}^{\prime} / T\right)\left(Z^{\prime-1}=\sum_{i} \exp \left(-E_{i}^{\prime} / T\right)\right)$. The modified molecular field CEF energies $E_{i}^{\prime}$ and eigenstates $\left|\psi_{i}^{\prime}\right\rangle$ are derived and discussed in Appendix B.

\section{A. Order parameter and saturation moment}

Calculating the diagonal (elastic) matrix elements of $J_{z}$ within the mf eigenstates $\left|\psi_{i}^{\prime}\right\rangle$ the selfconsistency equation of the order parameter may be given as

$$
\left\langle J_{z}\right\rangle_{T}=2 \sum_{i} p_{i}^{\prime}\left[\beta u_{i} w_{i}+v_{i}\left(\alpha u_{i}+\tilde{\alpha} w_{i}\right)\right] .
$$

The primed quantities generally refer to the $\mathrm{mf}$ values in the ordered state with nonvanishing $\left\langle J_{z}\right\rangle$. The latter appears

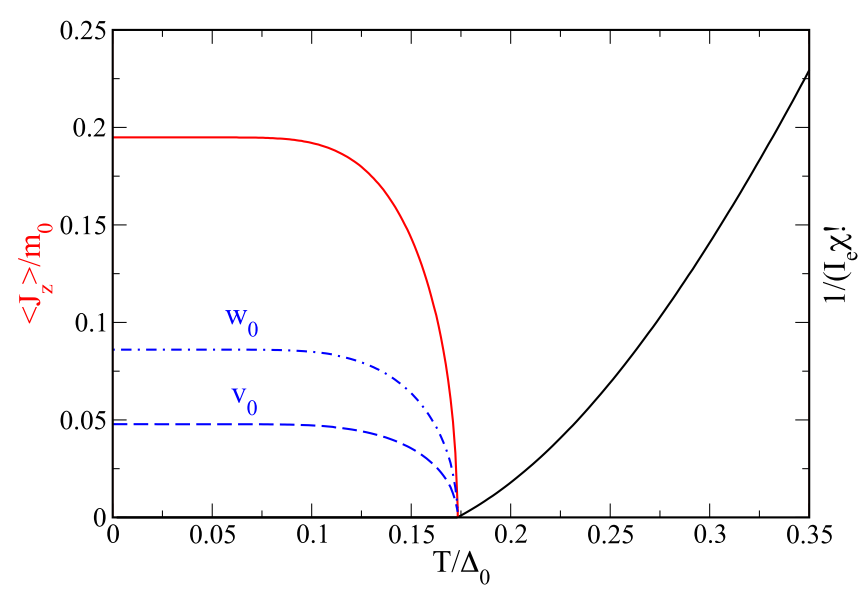

FIG. 7. Temperature dependence of inverse normalized susceptibility in the paramagnetic phase (black) and order parameter (induced moment $\left\langle J_{z}\right\rangle$ ) in the AF phase (red) normalized to $m_{0}=$ $\left(\alpha^{2}+\beta^{2}\right)^{\frac{1}{2}}$. The transition for $\xi_{s}=1.02$ and $\xi_{a}=-0.2$ is of second order (Fig. 5). The moment appears due to the mixing of excited $|1\rangle$ and $|2\rangle$ into the mf ground state $\left|\psi_{0}\right\rangle$ [Eq. (B4)]. Their coefficients $v_{0}, w_{0}$ are shown as broken blue lines[scheme as Fig. 2(a)].

implicitly in Eq. (50) through the mf energy levels $E_{i}^{\prime}$ and the coefficients $\left(u_{i}, v_{i}, w_{i}\right), i=0,1,2$ of the wave functions $\left|\psi_{i}^{\prime}\right\rangle$. The resulting temperature dependence of the AF order parameter $\left\langle J_{z}\right\rangle$ below $T_{m}$ together with the paramagnetic inverse static RPA susceptibility $\chi^{-1}(0, T)$ above $T_{m}$ is shown in Fig. 7 for a value of $\xi_{a}<1$ that results in a second-order transition. The divergence of $\chi(0, T)$ at $T_{m}$ triggers the appearance of the induced moment $\left\langle J_{z}\right\rangle$. The latter is due to the mixing of excited $|1\rangle,|2\rangle$ CEF states into the $\mathrm{mf}$ ground state $\left|\psi_{0}\right\rangle$ [Eq. (B4)]. The figure also displays the $T$ dependence of self-consistent admixture coefficients $v_{0}, w_{0}$ of excited states $|1\rangle,|2\rangle$ into the molecular field ground state $\left|\psi_{0}^{\prime}\right\rangle$ according to Eq. (B4). In contrast, the similar Fig. 8 presents the case of the first-order transition $\left(\xi_{a}>1\right)$ for two different $T_{m}$.

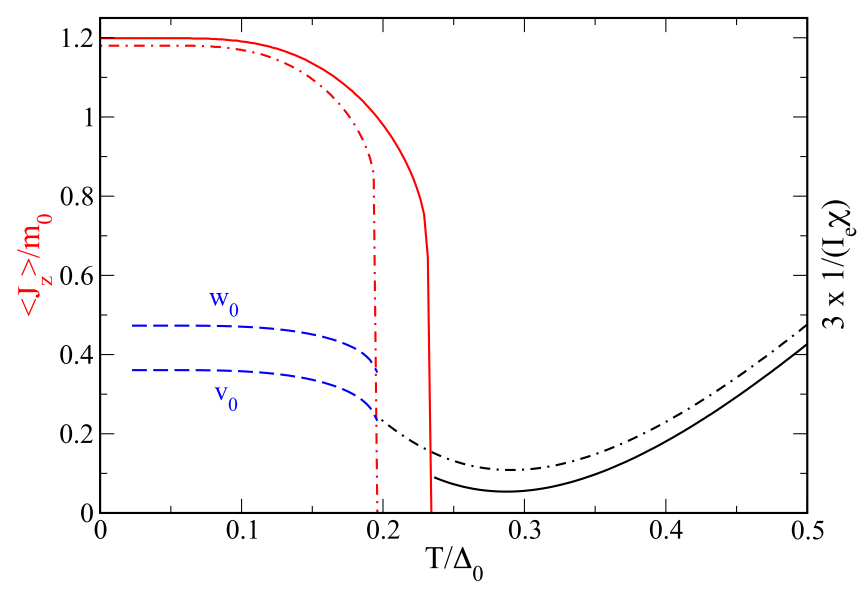

FIG. 8. Same as Fig. 7, now for $\xi_{s}=0.85,0.83$ (full and broken black/red lines) and $\xi_{a}=2.5$ when the transition is of first order (Fig. 6) with jump in $\left\langle J_{z}\right\rangle$. Note that susceptibility above $T_{m}$ does not diverge due to first-order character. Ground-state admixture coefficients $v_{0}, w_{0}$ (broken blue lines) also jump to finite values at $T_{m}$ [scheme as Fig. 2(b)]. 


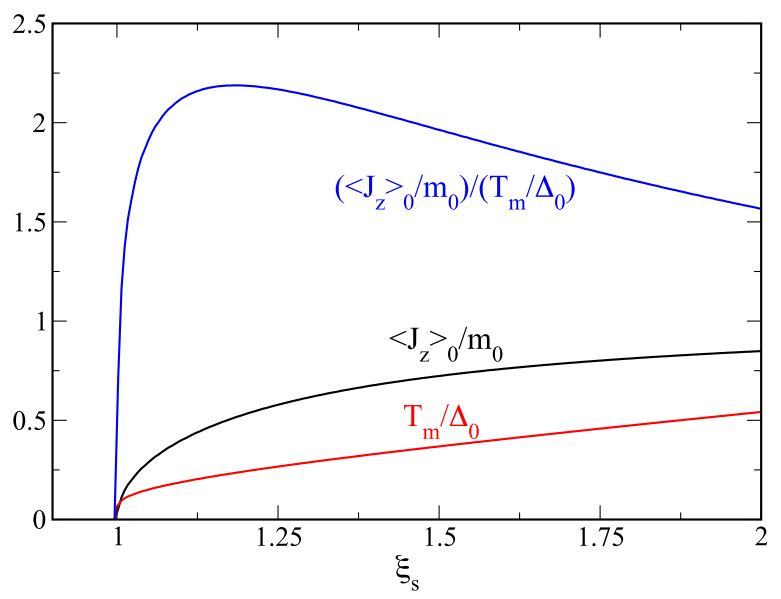

FIG. 9. Normalized saturation moment $\left\langle J_{z}\right\rangle_{T=0} / m_{0}$ with $m_{0}=$ $\left(\alpha^{2}+\beta^{2}\right)^{\frac{1}{2}}$, transition temperature $T_{m} / \Delta_{0}$, and their ratio as function of $\xi_{s}$. Matrix elements $\alpha, \beta, \tilde{\alpha}$ as in Fig. 7 for weakly symmetric case with $r=1$. Here the interaction constant $\mathrm{I}_{e}$ is varied leading to concomitant variation of $\xi_{s}$ and $\xi_{a}$. The ratio shows steep decrease close to quantum critical point [scheme as Fig. 2(a)].

There the susceptibility $\chi(0, T)$ no longer diverges at $T_{m}$ and the order parameter $\left\langle J_{z}\right\rangle$ and admixture coefficients jump to a finite value. From tracing $\left\langle J_{z}\right\rangle=0$ for different $\xi_{s}$, the firstorder transition line in the inset of Fig. 6 (red symbols) may be obtained.

The saturation moment at zero temperature is obtained from Eq. (50) as

$$
\left\langle J_{z}\right\rangle_{T=0}=2 u_{0}\left(\alpha v_{0}+\beta w_{0}\right)
$$

where on the r.h.s the index zero refers to the ground state $\left|\psi_{0}^{\prime}\right\rangle$. For the TSM, this equation cannot be solved explicitly for $\left\langle J_{z}\right\rangle$ since the latter enters on the r.h.s in a complicated manner in the mixing coefficients and associated $\mathrm{mf}$ energies (see Appendix B). As a reference, we give the expression for the two-singlet case (discarding the state $|2\rangle$ for the moment) where it can be derived [14] explicitly as

$$
\left\langle J_{z}\right\rangle_{T=0}=\alpha \frac{1}{\xi_{\alpha}}\left(\xi_{\alpha}^{2}-1\right)^{\frac{1}{2}}=\left\{\begin{aligned}
\alpha & \xi_{\alpha} \gg 1 \\
\alpha(2 \delta)^{\frac{1}{2}} & \xi_{\alpha} \simeq 1+\delta .
\end{aligned}\right.
$$

Thus the saturation moment and its ratio to the transition temperature $\left(\left\langle J_{z}\right\rangle_{T=0} / \alpha\right) /\left(T_{m} / \Delta\right)=(2 \delta)^{\frac{1}{2}} \ln \left(\frac{2}{\delta}\right) \rightarrow 0$ vanish when the induced magnet is close to the quantum critical point, i.e., $\delta \rightarrow 0$. This is in marked contrast to a conventional semiclassical (degenerate $S=\frac{1}{2}$ ) magnet [1] where the corresponding ratio is constant, given by $\left\langle S_{z}\right\rangle_{T=0} /\left(T_{m} / I_{e}\right)=1$ in that case. This peculiar dependence of saturation moment and its ratio with the transition temperature on the control parameters is also apparent in the TSM [Eq. (51)] as presented in Fig. 9. The saturation moment (now normalized to $m_{0}=$ $\left.\left(\alpha^{2}+\beta^{2}\right)^{\frac{1}{2}}\right)$ increases with square-root-like behavior above the critical parameter $\xi_{s}^{c}=1$ approaching unity for $\xi_{s} \gg 1$. Because $T_{m}$ varies only logarithmically for $\xi_{s} \rightarrow \xi_{s}^{c}$ the ratio of both quantities (blue line) first increases and the rapidly drops to zero.

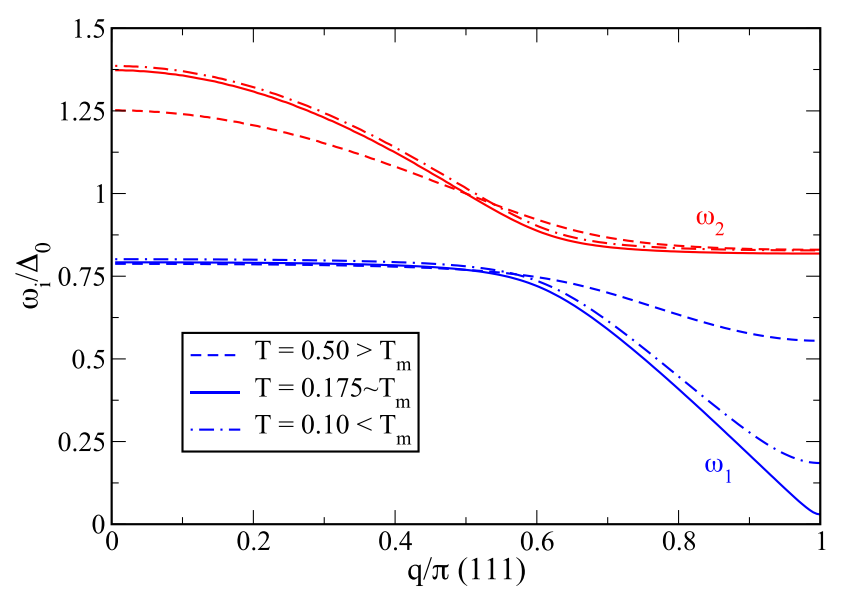

FIG. 10. Exciton dispersions for three temperatures above, equal and below the AF transition temperature at $T_{m} \simeq 0.175$. The softening of the critical mode $\omega_{2}(\mathbf{q})$ at the zone boundary AF point $\mathbf{Q}=(\pi, \pi, \pi)$ is reversed below $T_{m}$ into a hardening with decreasing temperature (cf. Fig. 11). Here we have above-critical values $\xi_{s}=1.02, \xi_{a}=-0.2$ [scheme as Fig. 2(a)].

\section{B. Collective excitations in the AF phase}

With $\left\langle J_{z}\right\rangle$ determined, we now may compute the renormalized excitation spectrum in the RPA approach in the induced moment phase. For this purpose, we need the renormalized local CEF energy differences $E_{i j}^{\prime}=E_{i}^{\prime}-E_{j}^{\prime}$ of molecular field states [Eqs. (B2)] and the inelastic matrix elements between them which lead to renormalized matrix elements $\alpha^{\prime}, \beta^{\prime}, \tilde{\alpha}^{\prime}$, which are now generally all nonvanishing because $\Theta$ is broken (Appendix B). In addition, we define modified effective temperature-dependent parameters $\alpha_{T}^{\prime 2}=$ $\alpha^{\prime 2} p_{01}^{\prime}, \beta_{T}^{\prime 2}=\beta^{\prime 2} p_{02}^{\prime}, \tilde{\alpha}_{T}^{\prime 2}=\tilde{\alpha}^{\prime 2} p_{12}^{\prime}$ analogous to Eqs. (13). With the replacements $\left(\Delta, \Delta_{0}, \tilde{\Delta}\right) \rightarrow\left(E_{10}^{\prime}, E_{20}^{\prime}, E_{21}^{\prime}\right)$ and $\left(\alpha_{T}^{2}, \beta_{T}^{2}, \tilde{\alpha}_{T}^{2}\right) \rightarrow\left(\alpha_{T}^{\prime 2}, \beta_{T}^{\prime 2}, \tilde{\alpha}_{T}^{\prime 2}\right)$, the exciton mode frequencies in the induced AF ordered phase may be obtained from Eqs. (12) and (16) by substitution.

An example of the temperature dependence of the exciton dispersions $\omega_{1,2}$ is presented in Fig. 10 using the parameter set of Fig. 7 (second-order case) for temperatures above, at, and below $T_{m}$. The flat mode $\omega_{3}$ in Fig. 3 which has vanishing intensity is not shown here. The $\omega_{1}$ dispersion displays the typical soft-mode behavior when temperature is lowered down to $T_{m}$ (dashed, full lines). However, immediately below $T_{m}$, the dispersion shifts to finite frequency again (dash-dotted).

The corresponding continuous temperature dependence for the $\mathbf{Q}=(\pi \pi \pi)$ soft mode with the same parameter set and another subcritical one for comparison is shown in Fig. 11. In the latter (broken lines), the zone boundary $\omega_{1}(\mathbf{Q})$ mode softens but then stays flat with lowering temperature while the upper mode $\omega_{2}(\mathbf{Q})$ is practically constant, see also Figs. 3 and 4 . When $\xi_{s}$ is above critical value (as in Fig. 10), $\omega_{1}(\mathbf{Q})$ now actually hits zero, triggering the onset of AF order shown in Fig. 7. Once the molecular field $h_{e}$ becomes finite and increases the splittings between renormalized levels at $E_{i}^{\prime}$, the critical mode $\omega_{1}(\mathbf{Q})$ is again stabilized to finite frequencies already seen in Fig. 10 for $T<T_{m}$. On the other hand, the upper mode for the parameters used shows very little temperature effect. It is certainly possible to fine tune the parameters such 


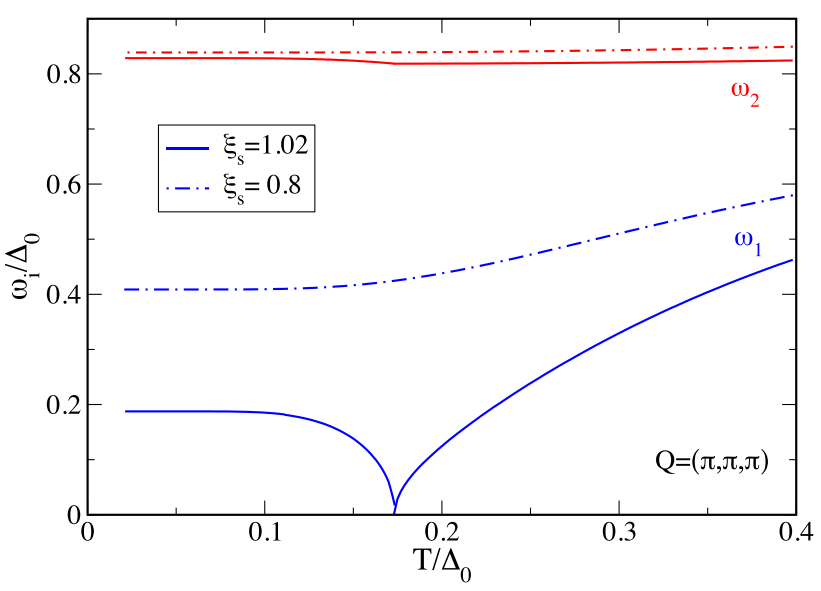

FIG. 11. Temperature dependence of zone boundary exciton modes $\omega_{1,2}(\mathbf{Q})$ for subcritical value $\xi_{s}=0.8$ (broken lines) and above-critical value $\xi_{s}=1.02$ ( $\xi_{a}=-0.2$ for both). The critical mode $\omega_{1}$ (connected with the $|1\rangle \leftrightarrow|2\rangle$ transition) softens at $T_{m}$ but rebounds immediately below. The upper mode is hardly affected by AF order (cf. Fig. 10) [scheme as Fig. 2(a)].

that both modes of the TSM become critical or closely so, but this seems rather artificial and, physically, one normally has to deal with just one critical mode as is the case, e.g., in the cubic singlet-triplet system $\mathrm{Pr}_{3} \mathrm{Tl}$ [9]. From the above discussion, it is clear that the softening of the critical mode in the case of a first-order transition is arrested at a finite energy value.

\section{SUMMARY AND CONCLUSION}

In this paper, we have given a complete survey of a most general extended TSM of induced moment quantum magnetism. It consists of three nonmagnetic CEF singlets coupled by nondiagonal matrix elements of one of the angular momentum components constrained by time reversal $\Theta$. Such low-lying TSM configurations occur frequently in rare earth or actinide compounds with $4 f^{2}$ or $5 f^{2}$ or other even occupation $f$-electron configurations. The model may be characterized by three individual $\left(\xi_{\alpha}, \xi_{\beta}, \xi_{\tilde{\alpha}}\right)$ but effectively two $\left(\xi_{s}, \xi_{a}\right)$ dimensionless control parameters, involving the CEF splittings, nondiagonal matrix elements, and intersite exchange.

We used two approaches to calculate the elementary excitation spectrum as a function of control parameters; the response function RPA formalism and the Bogoliubov quasiparticle approach. They agree on the basic properties of the magnetic exciton dispersions and their soft mode behavior as function of $\left(\xi_{s}, \xi_{a}\right)$. While the latter approach is only practical at low temperature ranges but gives the Bloch states of exciton bands, the RPA formalism covers all temperatures and, in particular, the mode softening as a function of temperature and the criticality condition for the onset of induced magnetism as function of $\left(\xi_{s}, \xi_{a}\right)$.

As an aspect of the TSM, we showed that for suitable control parameters a temperature-induced hybridization of modes takes place with an anticrossing of the two exciton dispersions resulting from excitations out of the ground state. On the other hand, a possible thermally excited mode stays dispersionless and is only visible at elevated temperatures.

The criticality condition leads to an equation for the dependence of ordering temperature on control parameters which may be solved explicitly for $T_{m}$ in the weakly and partly symmetric cases. For $\xi_{a}<1$, the condition for a finite induced ordering temperature is always given by $\xi_{s}>1$, independent of the values of $\xi_{a}$ and the splitting ratio r. Furthermore, in this case, the transition is always of second order as evidenced by the calculation of paramagnetic susceptibility and temperature-dependent order parameters.

Another possibility not observed in the singlet-singlet case arises for the phase diagram and magnetic ordering temperature $T_{m}\left(\xi_{s}, \xi_{a}\right)$ for $\xi_{a}>1$, which means that the thermally excited nondiagonal processes are important. Then the second-order transition at $T_{m}$ extends to control parameter $\xi_{s}<1$ and finally turns into a first-order transition at the critical point $T^{*}$, as demonstrated by the behavior of susceptibility and order parameter temperature dependence.

The latter is obtained from the mean-field self-consistency equations. The resulting molecular field enters into the dynamics via renormalized local CEF energies and nondiagonal matrix elements. Their influence leads to a resurgent stiffening of the soft mode immediately below $T_{m}$, which mimics the order parameter. The stiffening is continuous when the transition at $T_{m}$ is of second order and has a jumplike behavior for the first-order transitions.

These predicted features may play a role in real $\mathrm{Pr}$ and U-based singlet excitonic magnets and deserve further experimental investigations. This also refers to pressure experiments. The latter may change the CEF splittings and matrix elements, and hence the control parameters, and therefore may allow us to tune between the different phases found in this TSM investigation.

\section{APPENDIX A: EXAMPLE: THREE SINGLET MODEL FROM TETRAGONAL $\left(D_{4 h}\right) f^{2}(J=4)$ CEF STATES}

As a concrete example for tetragonal $D_{4 h}$ TSM, we discuss a TSM-level scheme derived from the $J=4$ ninefold degenerate total angular momentum multiplet relevant for $4 f^{2}$ and $5 f^{2}$ configurations. In the $D_{4 h}$ point group CEF environment, there are five singlets and two doublets [18,32]. This fact rests solely on the symmetry reduction of the total angular momentum representation of the full rotation group corresponding to $J=4$ to the $D_{4 h}$ representations $\Gamma_{1}^{(1)}, \Gamma_{1}^{(2)}, \Gamma_{2}$ [singlet group $\left.s_{1}(+1)\right], \Gamma_{3}, \Gamma_{4}$ [singlet group $s_{2}(-1)$ ], and (non-Kramers) doublets $\left.\Gamma_{5}^{(1)}, \Gamma_{5}^{(2)}(0)\right)$ where the number in parentheses indicates the character of the representation under $C_{4}$ rotation.

The wave functions and sequences of singlet and doublet energies in a concrete case are then to be obtained, in the simplest manner, by a local CEF Hamiltonian derived from a point charge model (PCM) [33,34] describing the crystal environment and expressed in standard Steven's operator technique. Thereby, the PCM parameters are commonly considered as free parameters to be determined from experiment (e.g., temperature dependence of susceptibility and INS peak positions and intensities). The energies $E_{i}$ and wave functions $|i\rangle[i=1 \ldots(2 J+1)]$ of CEF multiplets are then determined 
by the five $\left.B_{m n}(m n)=(20),(40),(44),(60),(64)\right)$ CEF parameters for $D_{4 h}$ symmetry.

For example, in $\mathrm{URu}_{2} \mathrm{Si}_{2}$, it was originally proposed by Santini and Amoretti [17] that the lowest states are the three singlets of group $s_{1}$. Explicitly, they are expressed in terms of $\left|J_{z}=M\right\rangle$ free ion states ( $z$ refers to the tetragonal axis) as

$$
\begin{aligned}
\left|\Gamma_{1}^{(1)}\right\rangle & =\cos \theta_{c}|0\rangle+\sin \theta_{c} \frac{1}{\sqrt{2}}(|4\rangle+|-4\rangle), \\
\left|\Gamma_{1}^{(2)}\right\rangle & =-\sin \theta_{c}|0\rangle+\cos \theta_{c} \frac{1}{\sqrt{2}}(|4\rangle+|-4\rangle), \\
\left|\Gamma_{2}\right\rangle & =\frac{1}{\sqrt{2}}(|4\rangle-|-4\rangle) .
\end{aligned}
$$

Within the PCM, their energies, referenced to the center of gravity of the three singlets, are given by [18]

$$
E\left(\Gamma_{1}^{(1,2)}\right)=\delta\left(\frac{1}{3} \pm \frac{1}{\cos 2 \theta_{c}}\right), \quad E\left(\Gamma_{2}\right)=-\frac{2}{3} \delta,
$$

where $\delta$ is a splitting parameter and $\theta_{c}$ a $\Gamma_{1}$ mixing parameter $\left(0 \leqslant \theta_{c} \leqslant \frac{\pi}{2}\right)$ both determined by the $B_{m n}$. This means within the local CEF-PCM, one should have two possible singlet (s1) sequences $|0\rangle-|1\rangle-|2\rangle$ given by (1) $\Gamma_{1}^{(1)}-\Gamma_{2}-\Gamma_{1}^{(2)}$ or inversely (2) $\Gamma_{1}^{(2)}-\Gamma_{2}-\Gamma_{1}^{(1)}$, depending on the size of $\theta_{c}$ and the sign of $\delta$. Therefore, $\Gamma_{2}$ should always lie between the two $\Gamma_{1}^{(1,2)}$ singlets. At the most, it can be accidentally degenerate with the lower $(\delta>0)$ or upper $(\delta<0) \Gamma_{1}^{(1,2)}$ singlet for $\theta_{c}=0, \frac{\pi}{2}$. Recent NIXS experiments [32] advocate that sequence 1 is realized with $\theta_{c} \simeq \pi / 2$. On the other hand, an alternative sequence with a $\Gamma_{2}$ ground state and $\Gamma_{1}^{(1,2)}$ excited singlets different from the simple CEF model has also been proposed from both experiments and DMFT theory $[26,35]$. In the above TSM, only $J_{z}$ has matrix elements and for sequence 1 they are given by (cf. Fig. 1)

$$
(\alpha, \beta, \tilde{\alpha})=\left(4 \sin \theta_{c}, 0,4 \cos \theta_{c}\right),
$$

and $\alpha, \tilde{\alpha}$ interchanged for sequence 2 . As in the orthorhombic case (Sec. II A), the dipolar matrix element between states with equal time-reversal symmetry (here $\Gamma_{1}^{(1,2)}$ with $\beta=$ $\left.\left\langle 0\left|J_{z}\right| 2\right\rangle\right)$ vanishes. Thus, staying strictly within the PCM, the tetragonal TSM can only support one of the possible dipolar excitation models shown Fig. 2(c). Therefore, the more flexible lower orthorhombic symmetry which should enable all cases in Fig. 2 has been chosen in Sec. II A.

\section{APPENDIX B: MOLECULAR FIELD ENERGIES, STATES AND MATRIX ELEMENTS IN THE INDUCED AF PHASE}

In this Appendix, we calculate the local mean-field energies, eigenstates, and matrix elements in the ordered $\mathrm{AF}$ phase characterized by a self-consistent order parameter $\left\langle J_{z}\right\rangle=$ $\left\langle J_{z}\right\rangle_{A}=-\left\langle J_{z}\right\rangle_{B}$ given by Eq. (50) where $A, B$ denote the AF sublattices. The total local Hamiltonian of Eq. (48) may be written explicitly as

$$
H_{\mathrm{CEF}}^{\mathrm{mf}}=\left(\begin{array}{ccc}
-\Delta & -\alpha_{e} & -\beta_{e} \\
-\alpha_{e} & 0 & -\tilde{\alpha}_{e} \\
-\beta_{e} & -\tilde{\alpha}_{e} & \tilde{\Delta}
\end{array}\right),
$$

with $\alpha_{e}=\alpha h_{e}, \beta_{e}=\beta h_{e}$ and $\tilde{\alpha}_{e}=\tilde{\alpha} h_{e}$. Here $h_{e}=I_{e}\left\langle J_{z}\right\rangle$ is the molecular field and we abbreviate $I_{e}=I_{e}(\mathbf{Q})=-z I_{0}>0$ with $\mathbf{Q}=(\pi, \pi, \pi)$ the $\mathrm{AF}$ ordering vector. The eigenvalues $E_{i}^{\prime}\left(h_{e}\right)$ of the molecular field Hamiltonian are then again given by the solutions of the cubic secular equations $(i=0,1,2)$ :

$$
\begin{aligned}
E_{i}^{\prime} & =2\left(\frac{|p|}{3}\right)^{\frac{1}{2}} \cos \left(\frac{\varphi}{3}+\varphi_{0}\right)-\frac{a}{3}, \\
\varphi & =\cos ^{-1}\left[-\frac{q}{2}\left(\frac{|p|}{3}\right)^{-\frac{3}{2}}\right],
\end{aligned}
$$

where $\varphi_{0}=\frac{2 \pi}{3}, \varphi_{1}=\frac{4 \pi}{3}, \varphi_{2}=0$, and $p=\frac{1}{3}\left(3 b-a^{2}\right) ; q=$ $\frac{2}{27} a^{3}-\frac{1}{3} a b+c$; with the cubic secular equation parameters defined by

$$
\begin{aligned}
& a=\Delta-\tilde{\Delta}, \\
& b=-\left[\Delta \tilde{\Delta}+\left(\alpha_{e}^{2}+\tilde{\alpha}_{e}^{2}+\beta_{e}^{2}\right)\right], \\
& c=\alpha_{e}^{2} \tilde{\Delta}^{2}-\tilde{\alpha}_{e}^{2} \Delta+2 \alpha_{e} \tilde{\alpha}_{e} \beta_{e} .
\end{aligned}
$$

We formally keep the last term in $c$ although it must vanish identically because one of the matrix elements has to be equal to zero due to time-reversal symmetry (Sec. II A). The phases $\varphi_{i}$ are denoted such that for the paramagnetic case with $h_{e}=0$ we recover $E_{i}^{\prime}=\hat{E}_{i}=-\Delta, 0, \tilde{\Delta}$ for $i=0,1,2$ consecutively, corresponding to the sequence in Fig. 1. The associated molecular field orthornormal eigenvectors are

$$
\left|\psi_{i}^{\prime}\right\rangle=u_{i}|0\rangle+v_{i}|1\rangle+w_{i}|2\rangle .
$$

These coefficients may be obtained for the general model by elimation from the eigenvalue equation $H_{\mathrm{CEF}}^{\mathrm{mf}}\left|\psi_{i}^{\prime}\right\rangle=E_{i}^{\prime}\left|\psi_{i}^{\prime}\right\rangle$. It is convenient to introduce the auxiliary factors

$$
\rho_{i}=\frac{\alpha_{e}\left(\tilde{\Delta}-E_{i}^{\prime}\right)+\tilde{\alpha}_{e} \beta_{e}}{E_{i}^{\prime}\left(\tilde{\Delta}-E_{i}^{\prime}\right)+\tilde{\alpha}_{e}^{2}}, \quad \tilde{\rho}_{i}=\frac{\beta_{e} E_{i}^{\prime}-\tilde{\alpha}_{e} \alpha_{e}}{E_{i}^{\prime}\left(\tilde{\Delta}-E_{i}^{\prime}\right)+\tilde{\alpha}_{e}^{2}} .
$$

Then the coefficients of the mf eigenfunctions are given by

$$
u_{i}=\left(1+\rho^{2}+\tilde{\rho}^{2}\right)^{-\frac{1}{2}}, v_{i}=-\rho_{i} u_{i} ; \quad w_{i}=\tilde{\rho}_{i} u_{i} .
$$

For the nondiagnonal matrix elements $\left\langle\psi_{i}\left|J_{z}\right| \psi_{j}\right\rangle(i \neq j)$, we obtain

$$
\begin{aligned}
& \alpha^{\prime}=\alpha\left(u_{0} v_{1}+v_{0} u_{1}\right)+\beta\left(u_{0} w_{1}+w_{0} u_{1}\right)+\tilde{\alpha}\left(v_{0} w_{1}+w_{0} v_{1}\right), \\
& \beta^{\prime}=\alpha\left(u_{0} v_{2}+v_{0} u_{2}\right)+\beta\left(u_{0} w_{2}+w_{0} u_{2}\right)+\tilde{\alpha}\left(v_{0} w_{2}+w_{0} v_{2}\right), \\
& \tilde{\alpha}^{\prime}=\alpha\left(u_{1} v_{2}+v_{1} u_{2}\right)+\beta\left(u_{1} w_{2}+w_{1} u_{2}\right)+\tilde{\alpha}\left(v_{1} w_{2}+w_{1} v_{2}\right) .
\end{aligned}
$$

We note that in the ordered state with $\Theta$ symmetry broken, all $\left(\alpha^{\prime}, \beta^{\prime}, \tilde{\alpha}^{\prime}\right)$ are nonzero due to the mixing of singlets by the molecular field, although one element of the set $(\alpha, \beta, \tilde{\alpha})$ must always vanish due to $\Theta$ symmetry in the nonmagnetic case. In the discussion of numerical results in Sec. VI, we restrict to the case $\alpha=0$ corresponding, e.g., to Fig. 2(b). From these matrix elements, the renormalized effective $T$-dependent matrix elements $\left(\alpha_{T}^{\prime 2}, \beta_{T}^{\prime 2}, \tilde{\alpha}_{T}^{\prime 2}\right)$ (see Sec. VI) which appear in the dispersions $\omega_{i}^{\prime}(\mathbf{q})$ and spectral function of $\chi_{0}\left(\mathbf{q}, i \omega_{n}\right)$ in the AF-ordered phase may be calculated in analogy to Eqs. (13). 
[1] N. Majlis, The Quantum Theory of Magnetism (World Scientific, Singapore, 2007).

[2] B. Schmidt and P. Thalmeier, Phys. Rep. 703, 1 (2017).

[3] B. Schmidt and P. Thalmeier, Phys. Rev. B 96, 214443 (2017).

[4] B. Schmidt and P. Thalmeier, New J. Phys. 17, 073025 (2015).

[5] J. Jensen and A. R. Mackintosh, Rare Earth Magnetism (Clarendon Press, Oxford, 1991).

[6] R. J. Birgeneau, J. Als-Nielsen, and E. Bucher, Phys. Rev. B 6, 2724 (1972).

[7] B. R. Cooper, Phys. Rev. B 6, 2730 (1972).

[8] D. B. McWhan, C. Vettier, R. Youngblood, and G. Shirane, Phys. Rev. B 20, 4612 (1979).

[9] W. J. L. Buyers, T. M. Holden, and A. Perreault, Phys. Rev. B 11, 266 (1975).

[10] T. M. Holden, E. C. Svensson, W. J. L. Buyers, and O. Vogt, Phys. Rev. B 10, 3864 (1974).

[11] P. Bak, Phys. Rev. B 12, 5203 (1975).

[12] J. G. Houmann, B. D. Rainford, J. Jensen, and A. R. Mackintosh, Phys. Rev. B 20, 1105 (1979).

[13] J. Jensen, K. A. McEwen, and W. G. Stirling, Phys. Rev. B 35, 3327 (1987).

[14] P. Thalmeier, Eur. Phys. J. B 27, 29 (2002).

[15] I. W. Sumarlin, J. W. Lynn, T. Chattopadhyay, S. N. Barilo, D. I. Zhigunov, and J. L. Peng, Phys. Rev. B 51, 5824 (1995).

[16] C. Broholm, H. Lin, P. T. Matthews, T. E. Mason, W. J. L. Buyers, M. F. Collins, A. A. Menovsky, J. A. Mydosh, and J. K. Kjems, Phys. Rev. B 43, 12809 (1991).

[17] P. Santini and G. Amoretti, Phys. Rev. Lett. 73, 1027 (1994).

[18] H. Kusunose and H. Harima, J. Phys. Soc. Jpn. 80, 084702 (2011).

[19] S. Kawarazaki, Y. Kobashi, M. Sato, and Y. Miyako, J. Phys. Condens. Matter 7, 4051 (1995).
[20] T. Naka, L. A. Ponomarenko, A. de Visser, A. Matsushita, R. Settai, and Y. Ōnuki, Phys. Rev. B 71, 024408 (2005).

[21] N. N. Tiden, E. S. Clementyev, P. A. Alekseev, E. V. Nefeodova, V. N. Lazukov, S. N. Gvasaliya, and D. Adroja, Physica B 378380, 1085 (2006).

[22] P. S. Savchenkov, E. S. Clementyev, P. A. Alekseev, and V. N. Lazukov, J. Magn. Magn. Mater. 489, 165413 (2019).

[23] R. Wawrzyńczak, B. Tomasello, P. Manuel, D. Khalyavin, M. D. Le, T. Guidi, A. Cervellino, T. Ziman, M. Boehm, G. J. Nilsen, and T. Fennell, Phys. Rev. B 100, 094442 (2019).

[24] G. H. Rao, Q. Huang, H. F. Yang, D. L. Ho, J. W. Lynn, and J. K. Liang, Phys. Rev. B 69, 094430 (2004).

[25] G. Zwicknagl, A. Yaresko, and P. Fulde, Phys. Rev. B 68, 052508 (2003).

[26] C. Haule and G. Kotliar, Nat. Phys. 5, 796 (2009).

[27] B. Grover, Phys. Rev. 140, A1944 (1965).

[28] Y.-L. Wang and B. R. Cooper, Phys. Rev. 172, 539 (1968).

[29] M. Rotter, M. D. Le, A. T. Boothroyd, and J. A. Blanco, J. Phys. Condens. Matter 24, 213201 (2012).

[30] A. Abragam and B. Bleaney, Electron Paramagnetic Resonance of Transition Ions (Clarendon Press, Oxford, 1970).

[31] P. Thalmeier, Europhys. Lett. 28, 507 (1994).

[32] M. Sundermann, M. Haverkort, S. Agrestini, A. A. Menovsky, Al-Zein, M. M. Sala, Y. Huang, M. B. M. Golden, A. de Visser, P. Thalmeier, L. H. Tjeng, and A. Severing, Proc. Natl. Acad. Sci. USA 113, 13989 (2016).

[33] M. T. Hutchings, Solid State Physics (Academic Press, New York, 1964), Vol. 16, Chap. 3, p. 227.

[34] K. R. Lea, M. J. M. Leask, and W. P. Wolf, J. Phys. Chem. Solids 23, 1381 (1962).

[35] H. H. Kung, R. E. Baumbach, E. D. Bauer, V. K. Thorsmolle, W.-L. Zhang, K. Haule, J. A. Mydosh, and G. Blumberg, Science 347, 1339 (2015). 